\title{
A novel practical state of charge estimation method: an adaptive improved ampere-hour method based on composite correction factor.
}

\author{
XIONG, X., WANG, S.-L., FERNANDEZ, C., YU, C.-M., ZOU, C.-Y. and \\ JIANG, C.
}

This is the peer reviewed version of the following article: XIONG, X., WANG, S.-L., FERNANDEZ, C., YU, C.-M., ZOU, C.-Y. and JIANG, C. 2020. A novel practical state of charge estimation method: an adaptive improved ampere-hour method based on composite correction factor. International journal of energy research, 44(14), pages 11385-11404, which has been published in final form at https://doi.org/10.1002/er.5758. This article may be used for non-commercial purposes in accordance with Wiley Terms and Conditions for Use of Self-Archived Versions. 


\section{INTERNATIONAL JOURNAL OF \\ ENERGY RESEARCH}

\section{A novel practical state of charge estimation method: an adaptive improved ampere-hour method based on composite correction factor.}

\begin{tabular}{|r|l|}
\hline Journal: & International Journal of Energy Research \\
\hline Manuscript ID & ER-20-16207.R1 \\
\hline Wiley - Manuscript type: & Research Article \\
\hline Date Submitted by the \\
Author: & $\mathrm{n} / \mathrm{a}$ \\
\hline Keywlete List of Authors: & $\begin{array}{l}\text { Xiong, Xin; Southwest University of Science and Technology, School of } \\
\text { Information Engineering } \\
\text { Wang, Shunli; Southwest University of Science and Technology, School } \\
\text { of Information Engineering } \\
\text { Fernandez, Carlos; Robert Gordon University } \\
\text { Yu, Chun-Mei; Southwest University of Science and Technology } \\
\text { Zou, Chuan-Yun } \\
\text { Jiang, Cong; Southwest University of Science and Technology, School of } \\
\text { Information Engineering }\end{array}$ \\
\hline & $\begin{array}{l}\text { Lithium-ion battery; state of charge; adaptive improved ampere-hour } \\
\text { method; composite correction factor }\end{array}$ \\
\hline
\end{tabular}

\section{SCHOLARONE Manuscripts}




\title{
A novel practical state of charge estimation method: an adaptive improved ampere-hour method based on composite correction factor
}

\author{
Xiong Xin ${ }^{\mathrm{a}}$, Shun-Li Wanga* ${ }^{\mathrm{a}}$, Carlos Fernandez ${ }^{\mathrm{b}}$, Chun-Mei Yuª , Chuan-Yun Zou ${ }^{\mathrm{a}}$, Jiang Cong ${ }^{\mathrm{a}}$ \\ ${ }^{a}$ School of Information Engineering, Southwest University of Science and Technology, Mianyang 621010, \\ China; ${ }^{b}$ School of Pharmacy and Life Sciences, Robert Gordon University, Aberdeen AB10-7GJ, UK.
}

\begin{abstract}
The research of real-time state of charge estimation method for lithium-ion battery is developing towards the trend of model diversification and algorithm complexity. However, due to the limitation of computing ability in the actual battery management system, the traditional ampere-hour method is still widely used. Firstly, temperature, charge-discharge current and battery aging are considered as the main factors that affecting the estimation accuracy of ampere-hour method under the condition that detection accuracy of the current sensor is determined. Secondly, the relationship between the state of charge and battery open-circuit voltage at different temperatures is analyzed, which is used to modify the initial state of charge. Thirdly, the influence mechanism of main factors on the effect of ampere-hour method is analyzed, and proposes a capacity composite correction factor to reflect the influence of charge-discharge efficiency, coulomb efficiency and battery aging comprehensively, and then update its value in real-time. Lastly, the adaptive improved ampere-hour formula and the complete state of charge estimation model is designed, and the estimation effect of this model is verified by comparing with other state of charge estimation methods in the experiment of dynamic cycle test. The results show that the estimation error of the adaptive improved method is less than $2 \%$ under two comprehensive working conditions, while the error of the traditional method is 5-10\%, and compared with extended kalman filter algorithm, it also gets a better state of charge estimation performance, which proves that this method is scientific and effective.
\end{abstract}

Key words: Lithium-ion battery; state of charge; adaptive improved ampere-hour method; composite correction factor *Corresponding author: Shun-Li Wang. Tel: +86-15884655563. E-mail address: 497420789@qq.com.

1. Introduction

State of charge (SOC) estimation of lithium-ion battery (LIB) is a core technology in battery management system (BMS) of electric vehicle (EV). Accurate SOC estimation can effectively enhance the safety and energy efficiency of batteries[1]. Lithium-ion battery is a complex non-linear system[2], complex working condition and sensor measurement process will produce inevitable noise, which makes accurate SOC estimation extremely difficult[3]. Plett defined SOC as a state quantity of physical meaning[4], but SOC of battery can't be measured by sensor directly, only by measuring other parameters such as voltage, current, resistance and temperature.

In the research field of state of charge estimation method for lithium-ion battery, according to the calculation 
complexity of the algorithm[5], it can be divided into two categories, simple estimation algorithm and complex algorithm[6]. Open-circuit voltage (OCV) method, ampere-hour (Ah) method and internal resistance method can estimate SOC by measuring the voltage, current or internal resistance of the battery singly[7]. However, these simple estimation methods have their limitations in the process of application[8]. For instance, the detection of open-circuit voltage and internal resistance requires that the battery is in thermodynamic equilibrium, which is not suitable for the online estimation of electric vehicles[9]. Although the Ah method is the most commonly used online state of charge estimation method, which method can achieve accurate SOC estimation[10], due to the current measurement noise and sampling error, the ampere-hour method will accumulate errors persistently and can't eliminate them by itself[11]. Therefore, the improvement ideas originate mainly from the problems that can't be solved by the above methods.

The first one is the model-based estimation method[12]. This kind of algorithm establishes the equivalent circuit model according to the empirical knowledge of battery reaction mechanism, and use the results of the model calculation voltage and the detection voltage to form the feedback[13], then obtains the error correction ability, such as extended kalman filter (EKF) algorithm and the unscented kalman filter algorithm[14], this algorithm has strong robustness and noise suppression ability. However, the accuracy of those model-based algorithms depends on the exact equivalent model, and usually, some parameters of the model vary significantly with the ambient temperature, charge-discharge current and battery aging[15]. For these reasons, many accurate equivalent models are proposed, such as the Thevenin model, PNGV model, second-order RC model and indefinite order $\mathrm{RC}$ model[16]. At the same time, the identification method of model parameters also develops from off-line to on-line parameter identification[17]. Unfortunately, due to the complexity of the electrochemical reaction of the battery itself and the operating condition of the battery[18], the model error always exists, which brings unavoidable errors to these model-based methods. In order to reduce the influence of model and measurement noise on the estimation accuracy, an adaptive extended kalman filter algorithm uses the noise covariance matching method to achieve the suppression of different initial noise, and the method of using multiple filtering algorithms to estimate SOC also significantly reduces the error caused by model noise[19] However, the improvement in SOC estimation accuracy caused by complex filtering algorithms may be far lower than the great increase of computation complexity[20]. Besides, with the development of artificial intelligence technology, the data-driven estimation method based on the black-box model is used to estimate SOC of battery[21], such as support vector machine and neural network algorithm. This kind of method does not need to know the actual battery models parameters, only needs a lot of input (battery voltage, current, 
temperature and impedance, etc.) and output data ( $\mathrm{SOC}, \mathrm{SOH})$ to training, and intelligently recognizes the relationship between SOC and input parameters[22]. However, this algorithm still has a long way to go, because of its high complexity[23].

In order to improve the performance of SOC estimation without increasing the complexity of the estimation method, some improved method based on traditional ampere-hour method itself becomes a solution. At first, Li quantitatively analyzed the influence of initial SOC precision[24], battery coulomb efficiency, Charge-discharge efficiency and battery capacity on the traditional ampere-hour method. Then, open-circuit voltage method[25] and load voltage method[26] are proposed to correct the initial error of SOC, the accuracy and auto disturbance rejection ability of improved algorithm are not improved substantially[27], because the parameters in the integral formula(formula (1)) are greatly affected by the ambient temperature, charge-discharge ratio, and state of health[24]. Feng proposed an improved ampere-hour method[28], which considered the influence of the change of battery available capacity on SOC estimation under different temperatures, but false to consider the influence of battery discharge rate and $\mathrm{SOH}$. Zhao proposed an improved ampere-hour method based on the least square method to estimate the coulomb efficiency in real-time[29], which can reduce the error accumulation caused by the disturbance of external factors, but it increases the complexity of the ampere-hour method, and the estimation effect is not different from the traditional kalman filter algorithm[30]. Therefore, this paper changes the research direction, no longer regards the coulomb efficiency, charge-discharge efficiency as state variables, and main work is as follows :

Firstly, this paper further explores the influence mechanism of external factors on ampere-hour method, and simplifies the idea of enhancing the ampere-hour method by real-time estimation of maximum available capacity. Secondly, the capacity composite correction factor is innovatively proposed, which represents the actual maximum available capacity of the battery when it is affected by various main disturbance factors(here refers to temperature, Charge-discharge current, $\mathrm{SOH}$ ), and then an adaptive improved ampere-hour formula based on the capacity composite correction factor is proposed. Thirdly, in order to obtain a more accurate initial value of ampere-hour method, battery necessary static time is discussed, then two kinds of OCV methods are used to modified the initial SOC in different situations. Finally, the online state of charge estimation of LIB based on the adaptive improved ampere-hour method is presented. 


\section{Theoretical analysis}

\subsection{The Principle of Traditional Ampere-hour Method}

Ampere-hour method is one of the most commonly used methods of SOC estimation, compared with model-based SOC estimation methods, it is simple to implement in BMS. The real-time SOC value can be calculated by using the initial SOC value plus the charge or minus the discharge capacity[31]. According to the research of $\mathrm{Li}$, the principle formula for the ampere-hour method is shown in the formula (1)[24]:

$$
S O C_{t}=S O C_{0}-\frac{\eta_{1}}{\eta_{2} C} \int_{0}^{t} I d t
$$

Among them: $S_{0}$ is the initial SOC, which influences the accuracy of SOC greatly, usually calculated by OCV method[32]; $\eta_{1}$ is the Coulomb efficiency, indicating the relationship between actual discharge current and theoretical discharge current; $\eta_{2}$ is the charge-discharge efficiency of battery, which represents the ratio of discharge capacity to charge capacity of batteries[33]; $C$ is the total capacity of battery, and $\int_{0}^{t} I d t$ is the integration of discharge current of batteries on time $[0, t]$. Generally, when the traditional ampere-hour method is used to estimate SOC[34], the coulomb efficiency and Charge-discharge efficiency are regarded as a constant, and the total capacity $\mathrm{C}$ of the battery is also regarded as a constant after the capacity experiment. In fact, these parameters are affected by the comprehensive effects of environmental temperature, Charge-discharge efficiency and state of health, and show obvious time-varying characteristics[35]. At the same time, current measurement noise and discrete sampling will cause unavoidable errors. Therefore, it is necessary to adjust the values of these parameters in time and calibrate the initial value of SOC accurately[36].

\subsection{Improvement Strategy of Ampere-hour Method}

\subsubsection{Improvement of SOC Initial Value Correction}

Open-circuit Voltage (OCV) refers to the potential difference between positive and negative electrodes when the external circuit is disconnected[25]. After a certain period of shelving, the terminal voltage of the battery is approximately equal to the open-circuit voltage. There is a relatively fixed functional relationship between the OCV and SOC, as shown in Figure.1. 


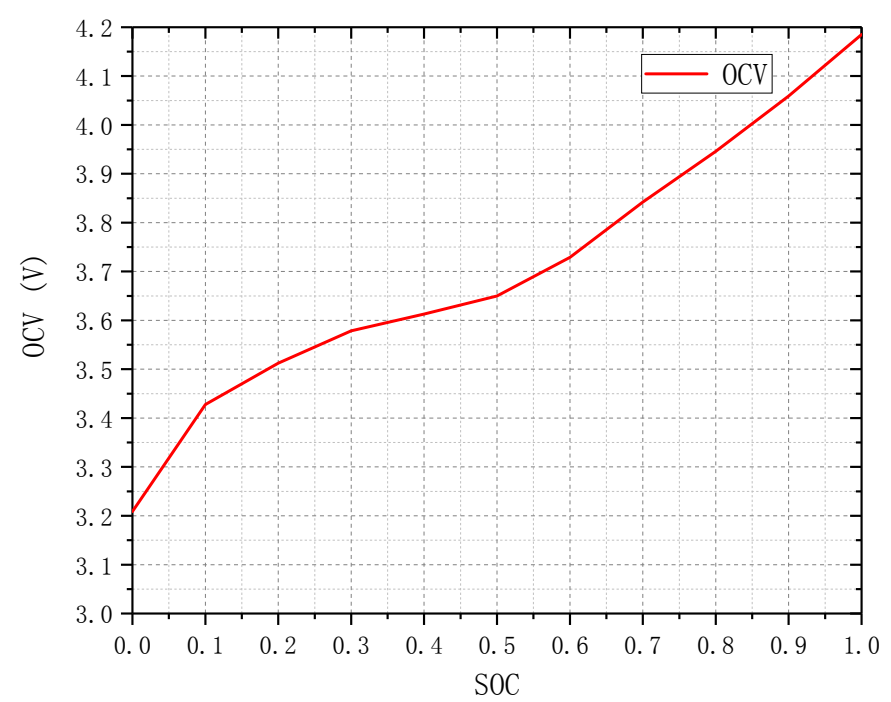

Fig. $1 \mathrm{OCV}-\mathrm{SOC}$ Curves at $25^{\circ} \mathrm{C}$

At a certain temperature and aging degree, the OCV of battery at each SOC point is measured, and then the OCV-SOC curve, which changes with SOC, is drawn. The corresponding functional relationship between OCV and SOC as shown in formula (2) can be obtained by curve fitting tools in MATLAB.

$$
O C V=f(S O C)
$$

There is a functional relationship between OCV and SOC of lithium-ion battery, so initial SOC can be calculated by measuring the OCV. According to the principle of SOC initial value modified by the open-circuit voltage method used in formula (1), updating and correcting of the initial SOC value depend on the corresponding relationship between OCV-SOC. However, the OCV-SOC relationship will be interfered by external factors under actual working conditions[37], resulting in SOC estimation error. Wu has studied the OCV curve at 40,30, 20, 10 and 0 degrees to describe a more accurate OCV-SOC curve, results show that the improved OCV method can elevate SOC estimation effects[38]. In addition, some papers have analyzed the shelf time of lithium-ion battery[39], considered that the necessary shelf time of the OCV-SOC curve is mainly affected by internal and external factors such as battery type, SOC status and temperature. Therefore, a shelving experiment was designed to explore the necessary time ( $\mathrm{x} /$ hour) for the battery terminal voltage return to a stable state at extreme temperature, and the data of $\mathrm{x}-\mathrm{SOC}$ and OCV-SOC were obtained through the shelving experiment. Then, use these data to calibrate SOC (0) can increase the accuracy of initial correction of SOC at extreme temperature.

However, when the shelve time of the battery can't meet the requirement of starting OCV method to modify 
the initial SOC for a long time, the SOC error caused by current sampling error will continue to accumulate, which will decrease the effects of Ah algorithm continually. Pei has developed a closed-circuit voltage method suitable for estimating SOC in real-time, and proved its effectiveness[26]. Therefore, in order to avoid the serious error of SOC estimation caused by the above situation, the closed-circuit voltage method is also used to correct the initial value of SOC.

\subsubsection{Coulomb efficiency analysis}

Coulomb efficiency can be understood as the ratio of actual discharge current to theoretical current, that is, the value of Coulomb efficiency determines the actual current. According to some literature[39], the influence of Coulomb efficiency on the estimation of SOC can also be reflected on the influence of discharge current on the actual capacity of batteries, as shown in Formula (3):

$$
\mathrm{SOH}, T, R \rightarrow \eta 1 \rightarrow \text { Current } \rightarrow \mathrm{SOC} \Leftrightarrow \mathrm{SOH}, T, R \rightarrow C \rightarrow \mathrm{SOC}
$$

The arrow in formula (3) indicates that the former determines or influences the latter, the bidirectional arrow represents an equivalent symbol. SOC is the abbreviation of state of charge, $\mathrm{SOH}$ indicates the state of health, $\mathrm{T}$ means ambient temperature, " $R$ " is the current ratio of charge and discharge[29], "Current" indicates the actual discharge current of the battery and " $\mathrm{C}$ " indicates the maximum usable capacity of the battery.

The significance of the formula (3) is that the effect of Coulomb efficiency $\eta 1$, which reflects the uncontrollable and unmeasurable aging decomposition of electrolytes and electrodes inside the battery, on the accuracy of the ampere-hour formula is shown in an equivalent way[40]. In order to simplify the ampere-hour formula, formula (3) is divided into the formula (4) and formula (5):

$$
\begin{gathered}
S O H \rightarrow \eta 1 \rightarrow \text { Current } \rightarrow S O C \Leftrightarrow S O H \rightarrow C \rightarrow S O C \\
R, T \rightarrow \eta 1 \rightarrow \text { Current } \rightarrow S O C \Leftrightarrow R, T \rightarrow C \rightarrow S O C
\end{gathered}
$$

The influence of the coulomb efficiency on SOC estimation by the ampere-hour method is reflected on two aspects. Formula (4) shows the decrease of coulomb efficiency caused by battery aging. The aging test is used to explore the aging attenuation law of battery capacity. Formula (5) shows the part of coulomb efficiency change caused by temperature and Charge-discharge current, and the SOC estimation error caused by temperature and Charge-discharge current is compensated by capacity calibration experiment of charge-discharge with different ratios at different temperature. Through the compensation strategy of the two aspects, the equivalent expression of coulomb efficiency is realized, and the difficulty of implementation of the adaptive improved ampere-hour method is weakened to a certain extent. 


\subsubsection{Charge-discharge efficiency analysis}

Charge-discharge efficiency refers to the ratio of discharge capacity $C_{\text {discharge }}$ to charge capacity $C_{\text {charge }}$ [38]. Usually, the charge-discharge efficiency of the battery is less than 1 due to the internal reaction and self-discharging which consumes part of the charge $C_{\text {loss }}$, as shown in Formula (6).

$$
\eta 2=\frac{C_{\text {discharge }}}{C_{\text {charge }}}=\frac{C_{\text {discharge }}}{C_{\text {discharge }}+C_{\text {loss }}}
$$

At present, the mainstream view is that $\eta_{2}$ is mainly affected by SOH and SOC of batteries. Similar to the compensation strategy of coulomb efficiency, the influence of temperature and Charge-discharge current on $\eta_{2}$ is also compensated by the capacity calibration experiment dominated by temperature and Charge-discharge current[41]. The process of the influence of $\eta_{2}$ on the estimation of SOC in Formula (1) can be briefly described in Formula (7).

$$
\mathrm{SOH} 、 \mathrm{SOC} \rightarrow \eta 2 \rightarrow \mathrm{C} \rightarrow \mathrm{SOC} \Leftrightarrow \mathrm{SOH} 、 \mathrm{SOC} \rightarrow \mathrm{C} \rightarrow \mathrm{SOC}
$$

The arrow in formula (7) indicates that the former determines or influences the latter. The influence of current $\mathrm{SOH}$ and SOC on the real-time SOC estimation of the formula (1) can't only be expressed with $\eta_{2}$, but also be reflected by the change of the actual available capacity "C", which is affected by SOH and SOC. In this way, the effect of current $\mathrm{SOH}$ and current SOC on equation (1) can be corrected by a particular calibration experiment of available capacity under different $\mathrm{SOH}$ and SOC states. Considering that the batteries are usually charged at a low state of charge, the Charge-discharge efficiency is close to 1 in this range of SOC[42]. In order to simplify the equation of the ampere-hour method, the influence of the current SOC on the estimation result of the ampere-hour Method was neglected.

\subsubsection{Actual Available Capacity analysis}

Calibrating the actual available capacity of battery under different influence factors can effectively modify its influence on the estimation of the ampere-hour method[43]. Considering that battery aging is a long-term process, the factors affecting the maximum usable capacity of battery are divided into two scales[44], then the cycle aging experiment and capacity calibration experiment under different temperatures and discharge ratios are designed to explore the mechanism of battery capacity change[45]. Based on the above theoretical and experimental analysis, an improved strategy with composite correction factor " $F$ " is proposed innovatively. The process of putting forward and calibrating the composite correction factor is shown in figure (2). 


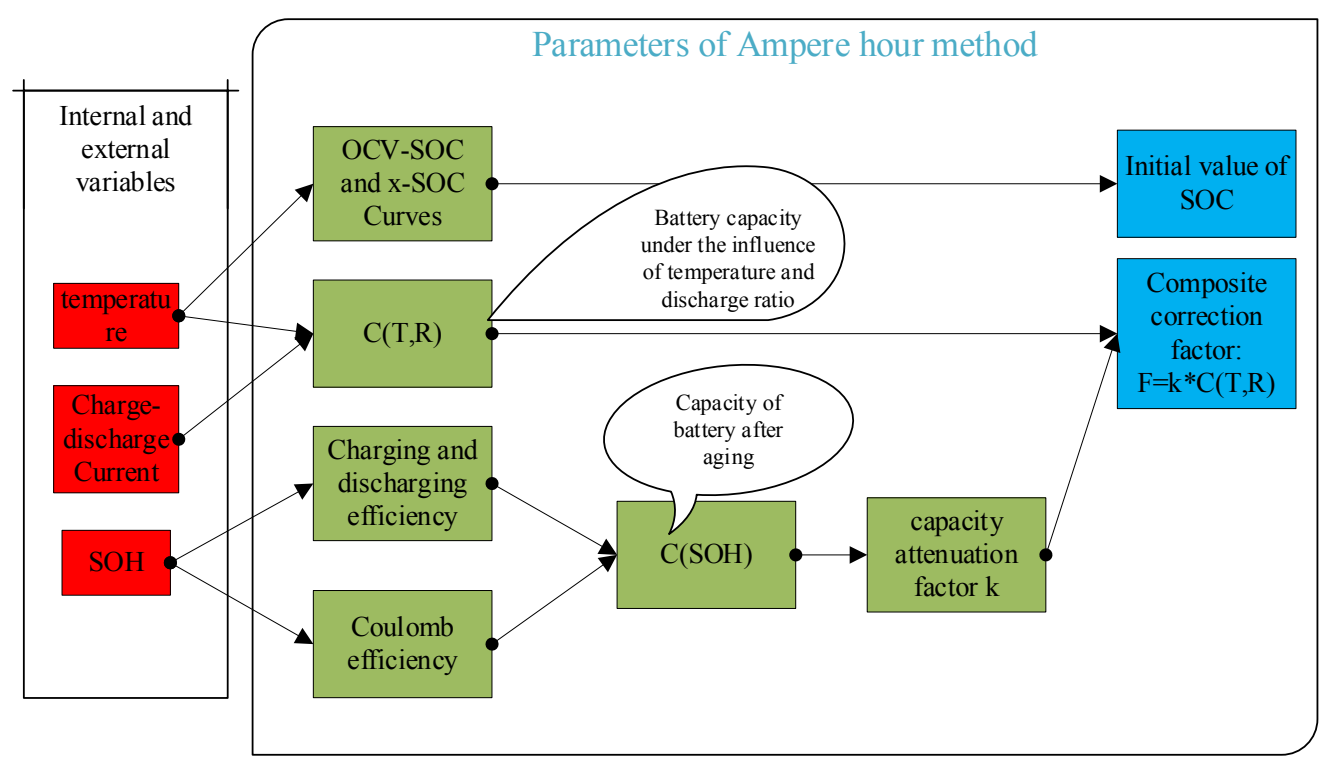

Fig. 2 Composite correction factor

Considering the influence of temperature, $\mathrm{SOH}$ and charge-discharge current on the specific parameters of ampere-hour method, $\mathrm{SOH}$ is taken as the main control factor of coulomb efficiency and charge-discharge efficiency, and capacity attenuation factor " $\mathrm{k}$ " is innovatively proposed to characterize the effect of coulomb efficiency and charge-discharge efficiency on the available capacity of the battery. Therefore, it is of great significance to calibrate the actual available capacity of battery under specific working conditions. Firstly, under the influence of $\mathrm{SOH}$, the calibration of available capacity effectively corrects the SOC estimation error caused by the change of Coulomb efficiency and charge-discharge efficiency. Secondly, the actual available capacity is calibrated at different temperatures and Charge-discharge current, which improves the effect of SOC estimation at extreme temperatures and different discharge ratio conditions and solves the problem of poor SOC estimation of battery at low temperature and high discharge rates.

\subsection{An Adaptive Improved Ampere-hour Algorithm}

\subsubsection{Adaptive Improved Ampere-hour Formula}

In Sections 2.1 and 2.2, analyses the merits and demerits of the traditional ampere-hour method and points out that the traditional ampere-hour method has insufficient accuracy in SOC estimation under the influence of extreme temperature, high rate discharge and $\mathrm{SOH}$ of battery. Feng studied the influence of the change of the actual available capacity of the battery on the SOC estimation under different temperatures[28], and proposed an improved ampere-hour integral formula as shown in formula (8):

$$
S O C(t)=S O C_{T}(t-1)-\eta \int_{t-1}^{t} \frac{I}{C_{T}} d t
$$

In formula (8), $\mathrm{SOC}(\mathrm{t})$ is $\mathrm{SOC}$ at the current temperature, $S O C_{T}(t-1)$ is the $\mathrm{SOC}$ converted from the previous 
time to the current temperature, $C_{T}$ is the capacity at different temperatures, and $\eta$ indicates Coulomb efficiency. This method considered the capacity change at different temperatures and the conversion from SOC at the previous temperature to SOC at the current temperature, which improves the performance of ampere-hour method in low temperature environment. However, the influence of $\mathrm{SOH}$ and discharge current on the SOC estimation can't be ignored[46]. Therefore, an adaptive improved ampere-hour method is proposed, which considers the changes of parameters of traditional ampere-hour method under the influence of three main factors of temperature, charge-discharge current and $\mathrm{SOH}$, the change rules of these parameters are further analyzed, and an improved ampere-hour method based on the composite correction factor is proposed. The adaptive improved ampere-hour integral formula is as follows:

$$
\operatorname{SOC}(\mathrm{t})=\operatorname{SOC}_{(T, R)}(0)-\frac{1}{k^{*} C(T, R)} \int_{0}^{t} I d t=S O C_{(T, R)}(0)-\frac{1}{F} \int_{0}^{t} I d t
$$

In formula (9), Combining the analysis of Figure 2 and formula (8), $\operatorname{SOC}_{(T, R)}(0)$ refers to the SOC converted to the current temperature and charge-discharge current from the last moment. During the iterative calculation of SOC, it is updated by assignment and conversion, but before each algorithm is started, it is corrected by the improved OCV method. $S O C(\mathrm{t})$ is SOC at the current temperature and current. $k$ is a capacity attenuation factor, usually between 0.8 and $1 . C(T, R)$ refers to the maximum available capacity at the current temperature and current, and "F"refers to the capacity composite correction factor. Using the adaptive improved ampere-hour algorithm shown in formula 9, the SOC estimation performance of the ampere-hour algorithm can be optimized in the following scenarios:

Considering the factors such as day-night temperature difference, seasonal temperature change and regional climate difference, the service temperature of lithium-ion batteries will be greatly affected. Considering that the average discharge current of urban electric vehicles varies frequently in actual use and considering that the internal parameters and various performances of lithium batteries will gradually decline with the increase of cycle charge-discharge times of lithium-ion batteries. Therefore, it is necessary to improve the traditional ampere-hour algorithm through the capacity composite correction factor.

\subsubsection{Adaptive Improved Ampere-hour Flowchart}

In order to apply the above adaptive improved Ah algorithm to the SOC estimation of actual lithium-ion batteries, a series of experiments need to be designed to explore the parameter variation rule in the formula (9), then a practical SOC estimation process is designed according to its estimation principle. Based on these dynamic parameters, a method of estimating SOC of lithium-ion batteries for BMS is proposed: an adaptive 
improved Ampere-hour method based on capacity composite correction factor, and its SOC estimation process is shown in figure 3.
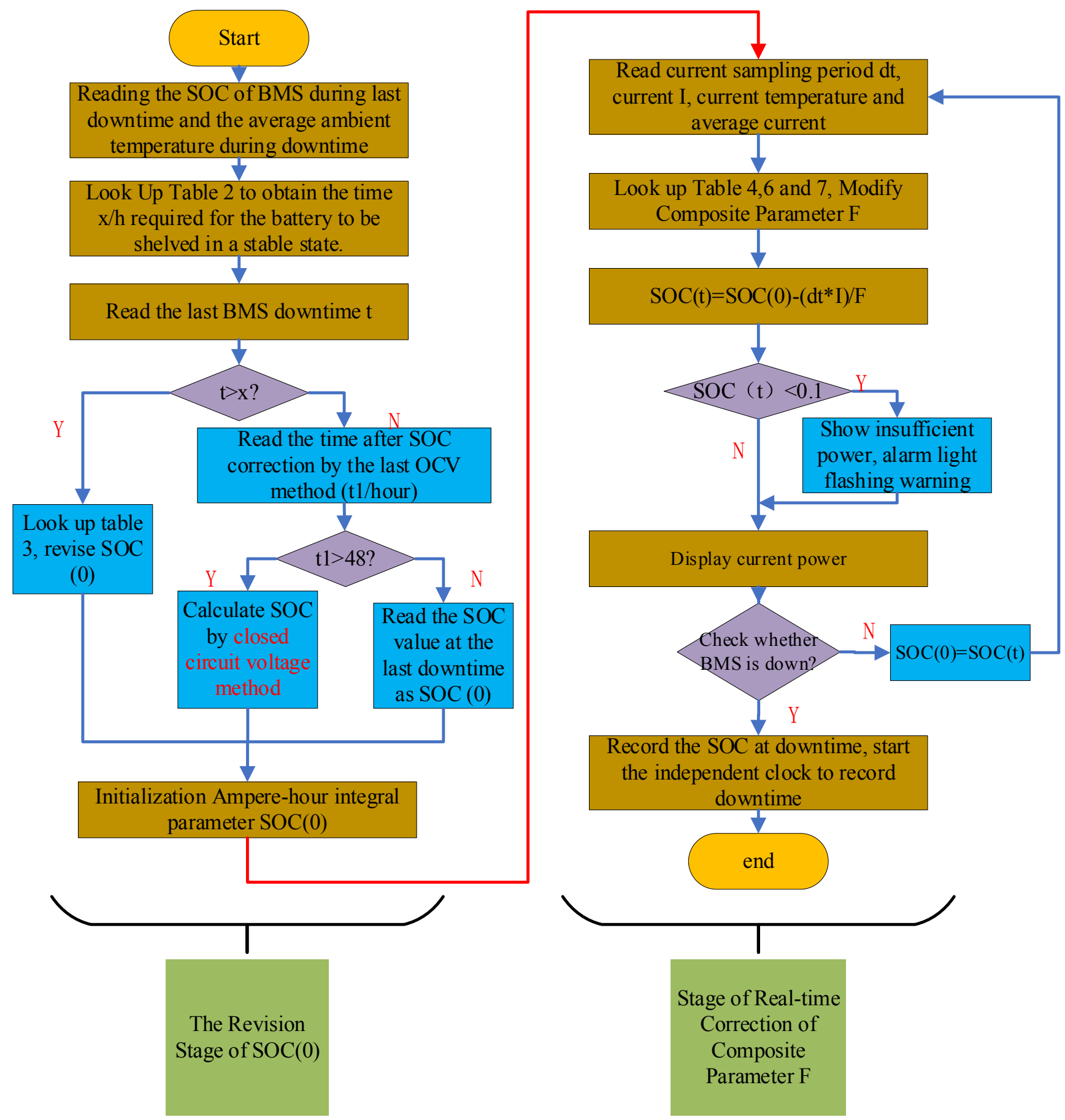

Fig. 3 An Adaptive Improved Ampere-hour Algorithms

At the beginning of the operation, the algorithm read the static time of BMS downtime ( $t$ ), read the SOC at last downtime, read the average temperature $(\mathrm{T})$ of batteries during downtime, read the time after SOC correction by the last OCV method (t1), and look up the $\mathrm{x}-(\mathrm{T}, \mathrm{SOC})$ curves of Table.2 to obtain the necessary time $\mathrm{x}$ at that time. If the downtime is longer than $\mathrm{x}$, the terminal voltage of the battery can be regarded as OCV, then the OCV - (SOC, T) curve of Table.3 is used to correct the initial SOC value. If less than $\mathrm{x}$, indicating that the 
battery is not stable very much[47], then judge that whether the battery has not carried out the OCV method to modify SOC value for a long time (48 hours), if it is not more than 48 hours, read the SOC value at the last downtime as SOC (0). Otherwise, use the closed-circuit voltage method to modify the SOC. The process of SOC calculation by the closed-circuit voltage method is as follows:

Step1: At first, the internal resistance "R" was identified based on the internal resistance model of LIB[26],

Step2: When the battery starts to run, take the sampled voltage $\left(U_{L}\right)$ and current $(I)$ into the following formula to estimate the current open-circuit voltage,

$$
O C V=U_{L}-I^{*} R
$$

Step3: Using the OCV calculated in step2 to get the corresponding SOC as the initial SOC of the next iteration.

In Figure.3, Table. 2 shows the relationship between $\mathrm{x}$ and SOC at downtime and average temperature during downtime. Table. 3 shows the relationship of OCV-SOC at different temperatures. Table.5, Table.6 and Table.7 show the relationship between composite correction factor F (intrinsic expression of Coulomb efficiency, charge-discharge efficiency and current available capacity) and internal and external factors of battery: SOH, temperature and charge-discharge current.

3. Experimental analysis

The experimental platform consists of ternary lithium-ion batteries $\left(\mathrm{LiNi}_{0.8} \mathrm{Co}_{0.2} \mathrm{O}_{2}\right)$, battery testing equipment (BTS200-100-10-4), thermostat (DGBELL) and an ordinary computer. The factory parameters of ternary lithium batteries are shown in Table.1.

Tab.1 Basic technical parameters of the battery

\begin{tabular}{|c|c|}
\hline Factor & parameter \\
\hline Size: length * ${ }^{*}$ width * height $/ \mathrm{mm}$ & $200 * 80 * 180$ \\
\hline Rated voltage $/ \mathrm{V}$ & 3.7 \\
\hline Maximum continuous load current $/ \mathrm{A}$ & $1 \mathrm{C}$ \\
\hline Charge cut-off voltage/V & 4.2 \\
\hline Discharge cutoff voltage $/ \mathrm{V}$ & 2.75 \\
\hline Working temperature $/{ }^{\circ} \mathrm{C}$ & $-30 \sim 70$ \\
\hline
\end{tabular}

The connection operation of the experimental equipment is shown in Figure.4. 


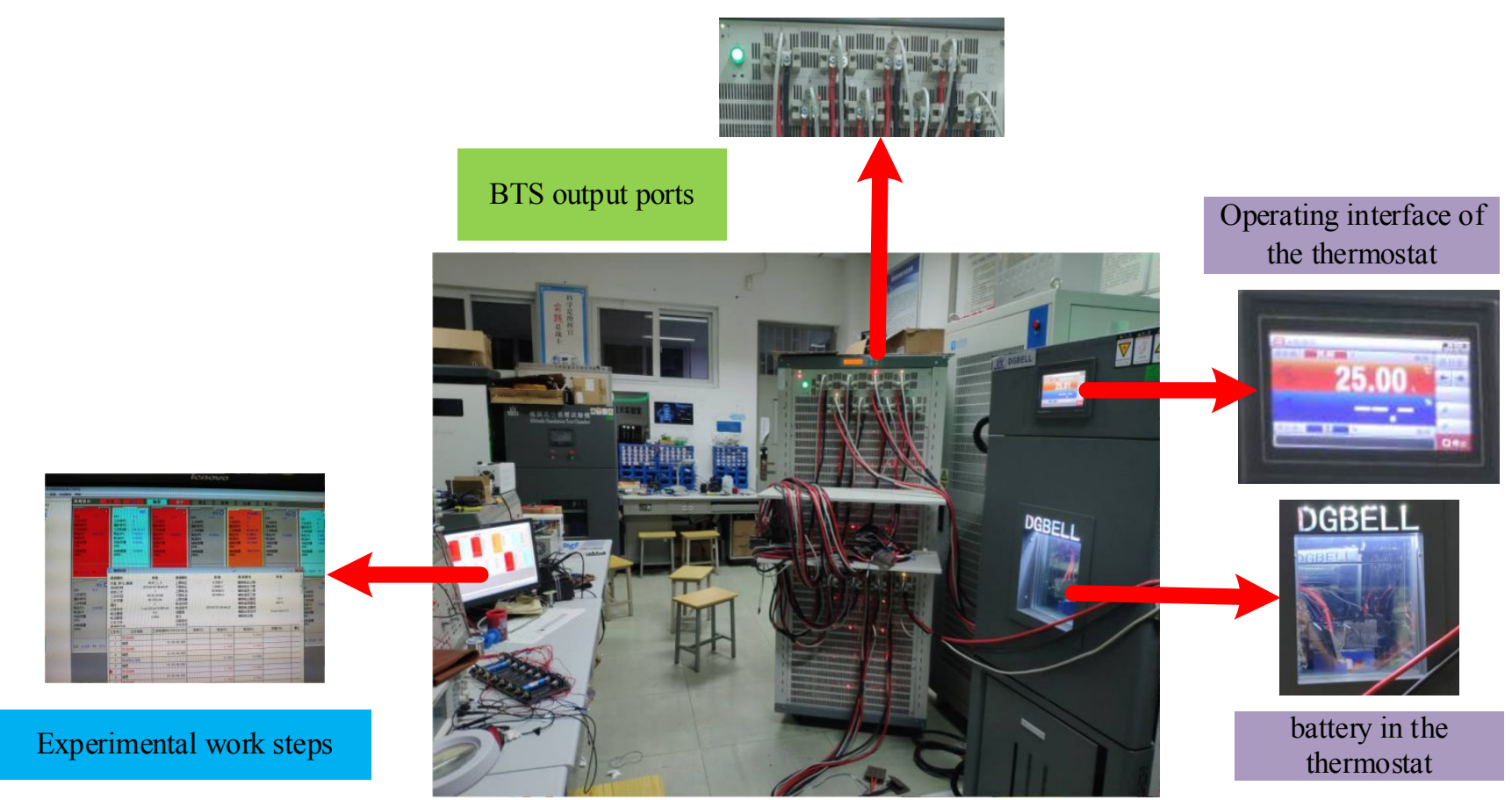

Fig. 4 Equipment Connection Diagram

The experimental lithium-ion battery is placed in the thermostat, and its positive and negative poles are connected with the corresponding channel lead-out wires of BTS (Battery Testing System). The BTS equipment communicates and transmits data to the computer through the network wires. The working mode of BTS is controlled by the professional management software of the BTS equipment on computer. Finally, the BTS device transfers and saves the detected experimental data to the computer. In the experiment, the thermostat is run for one hour to ensure that the battery is in the desired temperature range, then start the professional software of the BTS, set the corresponding working steps and protection conditions of the experiment, then run the experiment, export and analyze the experimental data from the professional software.

\subsection{Necessary shelf time exploratory experiment}

The shelf time of battery at different SOC points and different temperatures $(-20,10$ and 45 degrees) was investigated to obtain the necessary shelf time $\mathrm{x}$ to correct the initial SOC of batteries by using open-circuit voltage method. Among them, $\mathrm{x}$ refers to the time from the start of battery shelf to the rise rate of battery terminal voltage is less than $1 \mathrm{mV} / \mathrm{min}$. The experimental steps at 10 degrees are described as follows:

(1) Constant current and constant voltage charging. Firstly, the battery is charged with a constant current of $0.2 \mathrm{C}$ until the voltage at the battery terminal rises to $4.2 \mathrm{~V}$ for the first time, then it is charged with a constant voltage of $4.2 \mathrm{~V}$ until the detected current is reduced to $0.05 \mathrm{C}$ for the first time. At this time, $\mathrm{SOC}=1$ and assignment $\mathrm{i}=1$.

(2) $10 \%$ of the total capacity of constant current discharge. (Discharge at a constant current of 30A)

(3) Put aside for $2 * i$ hours to calculate the voltage rise rate for this period. 
(4) The shelf time is $\mathrm{x}$ when the voltage rise rate is less than $1 \mathrm{mV} / \mathrm{min}$ for the first time. Record the value of $\mathrm{x}$, that is, the necessary shelf time at this SOC point in the current temperature. If the voltage rise rate of this stage is not less than $1 \mathrm{mV} / \mathrm{min}$, then $\mathrm{i}=\mathrm{i}+1$, return to step (3). To determine whether SOC is less than 0.1 , step 2 is returned if SOC is not less than 0.1. If SOC is less than 0.1, the experiment is completed.

The detailed experimental procedure is shown in Fig. 5.

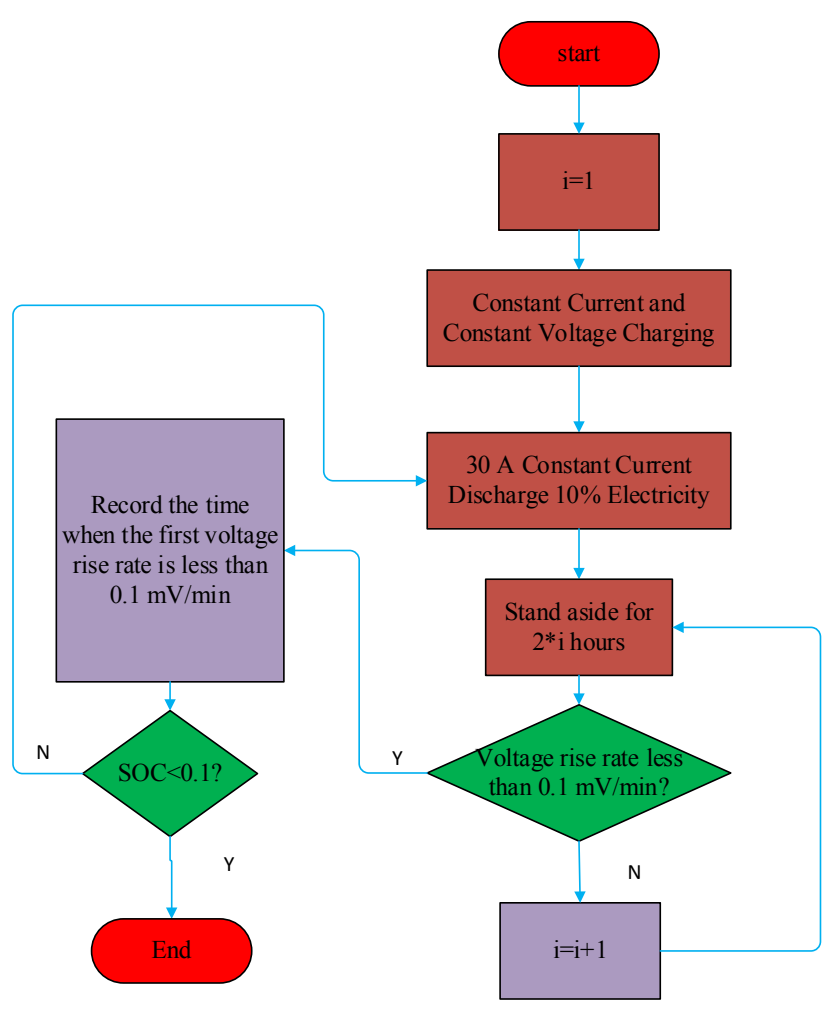

Fig. 5 Flowchart of necessary shelf time experiment

According to the experimental steps described in Fig. 5, the experiments were repeated at different temperatures. The experimental results are summarized in Table.2. When the temperature and SOC are given, the necessary shelf time $\mathrm{x}$ (hours) can be obtained by looking up Table.2.

Tab.2 Necessary shelf time $\mathrm{x}$

\begin{tabular}{|c|c|c|c|}
\hline SOC $\backslash$ Temperature & $-20^{\circ} \mathrm{C}$ & $10^{\circ} \mathrm{C}$ & $45^{\circ} \mathrm{C}$ \\
\hline 0.1 & 7.2 & 8.23 & 5.5 \\
\hline 0.2 & 5.5 & 7.52 & 4 \\
\hline 0.3 & 4.52 & 5.88 & 3 \\
\hline 0.4 & 3.85 & 5.102 & 2.5 \\
\hline 0.5 & 3.4 & 4.2 & 2.2 \\
\hline
\end{tabular}


1

\begin{tabular}{|c|c|c|c|}
\hline 0.7 & 1.55 & 2.78 & 1.8 \\
\hline 0.8 & 1.2 & 1.82 & 1.3 \\
\hline 0.9 & 0.89 & 1.1 & 1 \\
\hline
\end{tabular}

Using the data summarized in Table.2, the graph shown in Figure.6 is drawn.

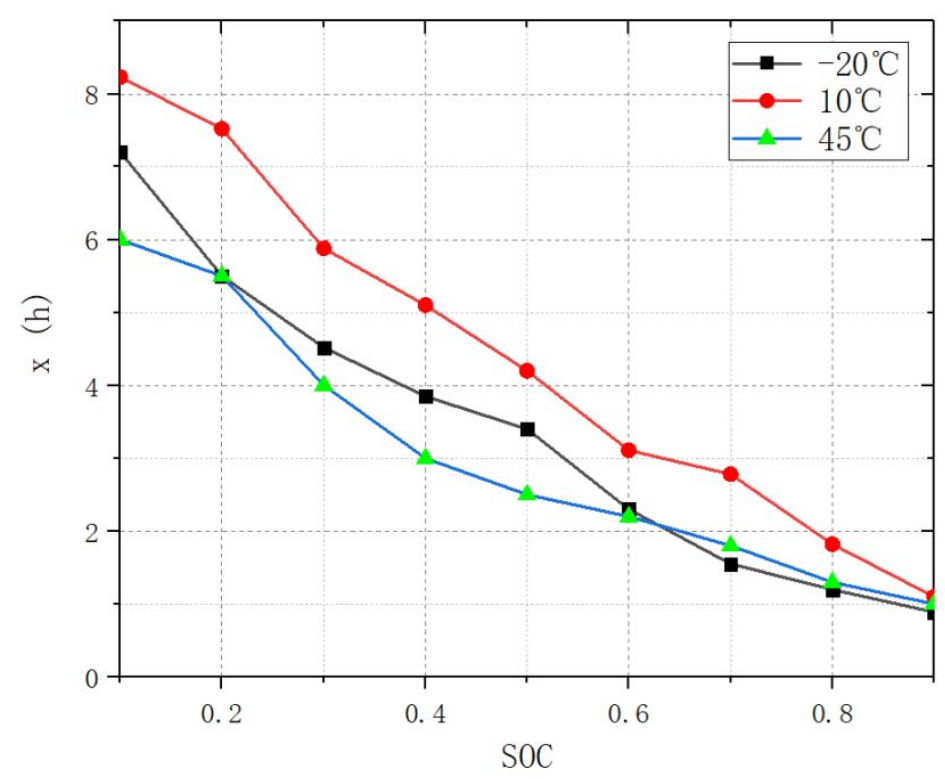

Fig. 6 x-SOC curves

The shelf time of ternary lithium-ion batteries is investigated, and the results show that SOC is inversely proportional to the time required to restore voltage stability at different temperatures. In the same SOC state, the necessary shelf time corresponding to different temperatures is different.

\section{2. $\quad$ OCV Experiment at Different Temperatures}

According to the experimental results of $\mathrm{x}$-SOC at different temperatures in 3.1 , an open-circuit voltage experiment at corresponding temperatures was designed. Because this experiment is relatively simple and similar to the necessary shelf time experiment in Section 3.1, the flow charts of OCV experiment at different temperatures are given only, as shown in Figure.7, without giving a detailed explanation of the experimental process. 


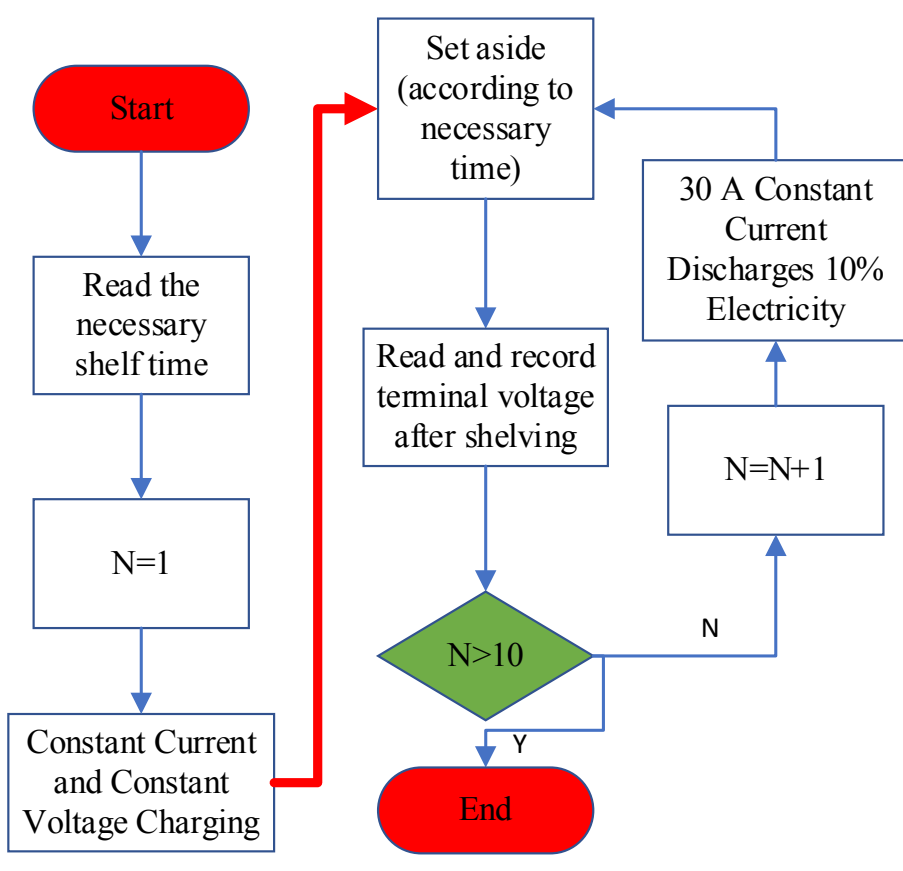

Fig. 7 Open-circuit voltage experiment flow

According to the flow chart shown in Figure.7, the open-circuit voltage is calibrated in each SOC with 0.1 intervals. The experimental data are shown in Table.3.

Tab.3 OCV-SOC at different temperatures

\begin{tabular}{|c|c|c|c|c|}
\hline SOC\Temperature & $-20^{\circ} \mathrm{C}$ & $0^{\circ} \mathrm{C}$ & $25^{\circ} \mathrm{C}$ & $45^{\circ} \mathrm{C}$ \\
\hline 1 & 4.1384 & 4.1567 & 4.1852 & 4.1750 \\
\hline 0.9 & 4.0012 & 3.9957 & 4.0590 & 4.0374 \\
\hline 0.8 & 3.8842 & 3.8726 & 3.9459 & 3.9142 \\
\hline 0.7 & 3.6913 & 3.7652 & 3.8420 & 3.7856 \\
\hline 0.6 & 3.5617 & 3.6425 & 3.7289 & 3.7134 \\
\hline 0.5 & 3.4924 & 3.6498 & 3.6498 & 3.6617 \\
\hline 0.4 & 3.4527 & 3.5721 & 3.6129 & 3.6014 \\
\hline 0.3 & 3.4013 & 3.5421 & 3.5785 & 3.3548 \\
\hline 0.2 & 3.3452 & 3.5133 & 3.5125 & 3.3016 \\
\hline 0.1 & 3.2342 & 3.4693 & 3.4278 & 3.2158 \\
\hline 0 & 3.0344 & 3.3401 & 3.2087 & 2.9600 \\
\hline
\end{tabular}

The experimental results are shown in Figure.8. 


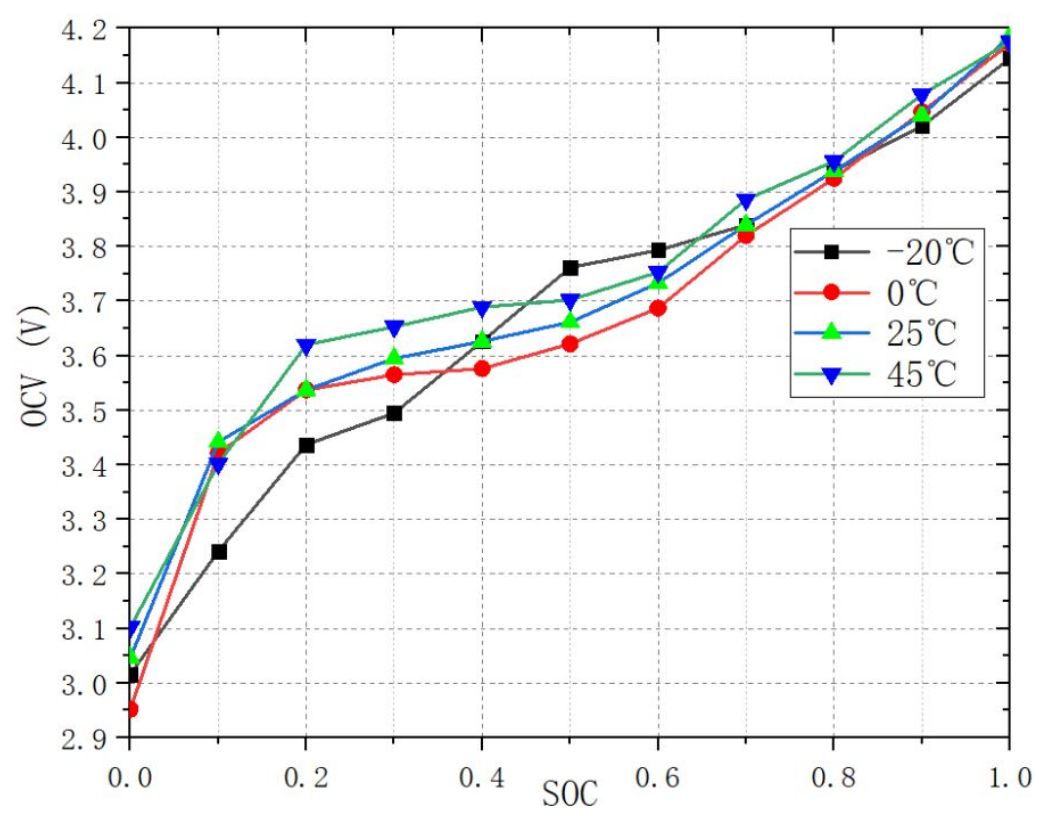

Fig. 8 OCV-SOC at Different Temperatures

According to the experimental results of Figure.8, The open-circuit voltage increases with the SOC at different temperatures, and the slope of the open-circuit voltage curve is obviously higher than that in the middle of the two sides of SOC. After calibrating the monotonous mapping relationship between OCV and SOC, the corresponding SOC can be calculated by measuring the steady-state OCV at different temperatures for SOC (0) correction.

\subsection{Aging experiment}

According to some papers, the aging experiment of ternary lithium-ion battery is designed[48]. The service life of battery is affected by many factors, such as the number of cycles, temperature and pressure. The purpose of aging experiment is to correct the influence of Coulomb efficiency and charge-discharge efficiency on the calculation of SOC by ampere-hour method. Therefore, the conventional aging experiments caused by the increase of recycling times at room temperature were designed, and the actual available capacity data of batteries under different recycling times were obtained. The experimental process is shown in Figure.9. Among them, the capacity test experiments are charged and discharged with standard current of $0.2 \mathrm{C}$, and the double discharging process is as follows:

(1) Discharging with constant current of $0.2 \mathrm{C}$ to cut-off voltage of $2.75 \mathrm{~V}$.

(2) Then shelving for 1 hour, discharging with constant current of $0.1 \mathrm{C}$ to cut-off voltage of $2.75 \mathrm{~V}$, which fully guarantees the discharge of battery.

Constant current and constant voltage charging:

(1) First $0.2 \mathrm{C}$ constant current charging to $4.2 \mathrm{~V}$ charging cut-off voltage.

(2) Then $4.2 \mathrm{~V}$ constant voltage charging until the current is reduced to $0.05 \mathrm{C}$.

Aging experiment was conducted with 1C charge and discharge, and the parameters of charge and discharge 
cut-off were consistent with the capacity test. It needs further explanation that this experiment mainly explores the relationship between the number of cycles used and the actual available capacity. The aging experiment has a temperature of 25 degrees and a Charge-discharge current of 1C. The capacity test is carried out 50 times per cycle until 300 cycles of Charge-discharge are completed.

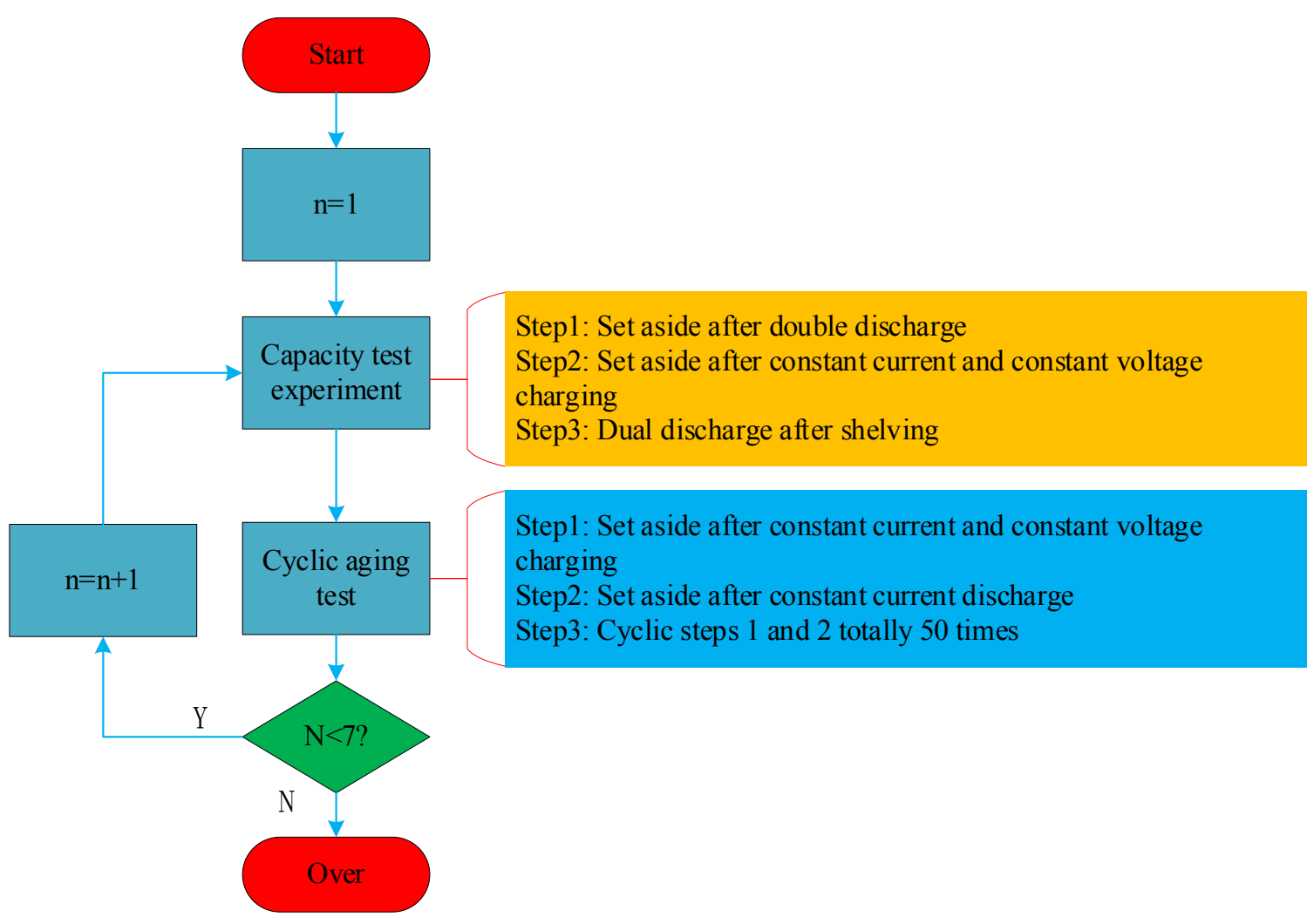

Fig. 9 Aging experimental process

After the above experiments, the actual available capacity of seven groups with different charge-discharge cycles was obtained, as shown in Table.4.

Tab.4 Discharge Capacity-Number of Cyclic Charges and Discharges

\begin{tabular}{|c|c|c|c|c|c|c|c|}
\hline $\begin{array}{c}\text { Number } \\
\text { of cycles }\end{array}$ & 0 & 50 & 100 & 150 & 200 & 250 & 300 \\
\hline Capacity & 46.40012 & 46.1021 & 45.8332 & 45.1453 & 44.6752 & 43.2412 & 42.3169 \\
\hline
\end{tabular}

According to the results of Table.4, the Dot-line graph shown in Figure.10 is drawn. 


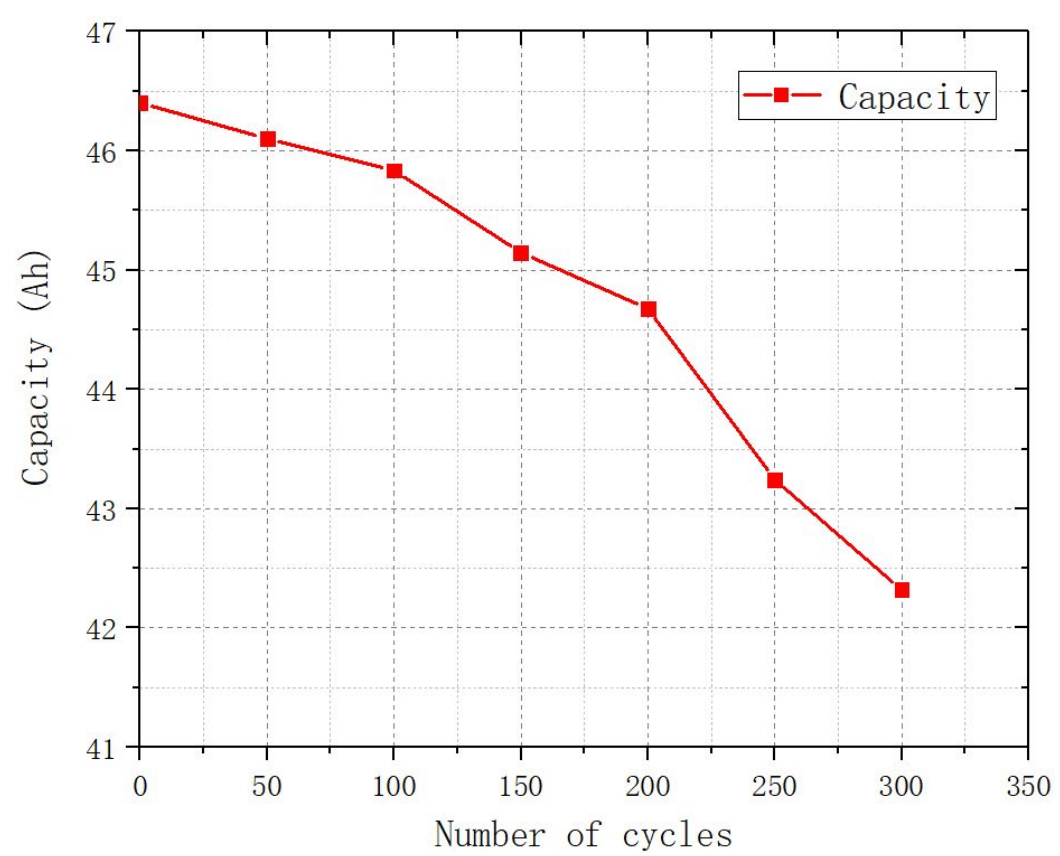

Fig. 10 Actual Available Capacity-Number of Cyclic Charges and Discharges

Curve fitting and its results. Based on the study of the relationship between the percentage of battery capacity attenuation and the number of charge-discharge cycles, a simplified capacity attenuation function is proposed on the basis of semi-empirical formula, as shown in formula (11).

$$
k=A^{*} \exp \left(-\frac{x}{B}\right)+C
$$

Formula (11) is a functional expression of capacity attenuation factor, in which $A$ and $B$ are constant values to be determined, $x$ represents the number of cycles of charge and discharge, $k$ represents the capacity attenuation factor. The capacity attenuation factor $k$ is fitted in MATLAB with the data in Table.4, and the curve fitting results are shown in Table.5.

Tab.5 Fitting Results of Capacity Attenuation Function

\begin{tabular}{|c|c|c|c|c|}
\hline parameter & A & $1 / B$ & C & R-square \\
\hline value & -0.8895 & -0.005825 & 47.33 & 0.9903 \\
\hline
\end{tabular}

The algebraic expression of capacity attenuation function can be obtained by least squares fitting of seven sets of data and introducing the three parameters fitted in Table.5 into Formula.7. The fitted function approximates the functional relationship between the actual available capacity of batteries and the number of cycles of charge and discharge, and it can be used to modify the capacity degradation of batteries caused by SOH in the adaptive improved Ampere-hour method. However, when the battery is used in practice, it is difficult to count the number of complete charge and discharge cycles, which leads to the misjudgment of the battery's real aging situation and the deviation of the actual capacity estimation. Therefore, a statistical method for the number of standardized battery cycles is 
proposed, in the 3.5.1 section, double discharge verification experiment is designed, standard charge and discharge times of the battery are calculated by this method, and the aging capacity of the battery is modified.

\subsection{Actual Available Capacity Calibration Experiments}

In order to quantify the influence of environment temperature and Charge-discharge current on battery capacity, capacity calibration experiments were designed under different temperature and Charge-discharge current. Five different charge-discharge capacity test experiments of $0.2 \mathrm{C}, 0.444 \mathrm{C}, 0.6 \mathrm{C}, 0.8 \mathrm{C}$ and $1 \mathrm{C}$ were designed under five temperature conditions of $-20,0,10,25$ and 45 .

The BTS channel wiring is introduced into the thermostat, connected to the two sides of battery, set the temperature parameters, and start the test when the fixed temperature value runs for 1 hour. By setting operation steps on BTS professional software, the output current and voltage of BTS can be controlled, and then the current and voltage loaded on both sides of the battery can also be controlled.

The experimental flow of capacity calibration at different temperatures and Charge-discharge currents is basically the same as that in the phase of capacity test of aging experiment in Figure.9, mainly due to the difference of ambient temperature and Charge-discharge currents. Therefore, it is no longer necessary to elaborate on the detailed capacity measurement test process.

The actual available capacity of batteries under two factors was obtained. Table.5 shows the charging capacity in constant current and constant voltage mode. According to the capacity calibration laboratory data obtained in Table.6, the dot-line graph shown in Figure.11 is drawn. According to the discharge capacity data in Table.7, the dot-line diagram shown in Figure.12 is drawn.

Tab.6 Charging capacity

\begin{tabular}{|c|c|c|c|c|c|}
\hline $\begin{array}{c}\text { Temperature\multiplie } \\
\mathrm{r}\end{array}$ & $0.2 \mathrm{C} \approx 9 \mathrm{~A}$ & $0.444 \mathrm{C} \approx 20 \mathrm{~A}$ & $0.6 \mathrm{C} \approx 27 \mathrm{~A}$ & $0.8 \mathrm{C} \approx 36 \mathrm{~A}$ & $1 \mathrm{C} \approx 45 \mathrm{~A}$ \\
\hline$-20^{\circ} \mathrm{C}$ & $31.537346 \mathrm{Ah}$ & $32.047580 \mathrm{Ah}$ & $33.585364 \mathrm{Ah}$ & $35.273583 \mathrm{Ah}$ & $38.521731 \mathrm{Ah}$ \\
\hline $0^{\circ} \mathrm{C}$ & $42.584253 \mathrm{Ah}$ & $42.323723 \mathrm{Ah}$ & $42.307856 \mathrm{Ah}$ & $42.268207 \mathrm{Ah}$ & $43.698293 \mathrm{Ah}$ \\
\hline $10^{\circ} \mathrm{C}$ & $44.829743 \mathrm{Ah}$ & $44.746519 \mathrm{Ah}$ & $44.691937 \mathrm{Ah}$ & $44.733196 \mathrm{Ah}$ & $44.769160 \mathrm{Ah}$ \\
\hline $25^{\circ} \mathrm{C}$ & $46.434770 \mathrm{Ah}$ & $46.259540 \mathrm{Ah}$ & $46.191140 \mathrm{Ah}$ & $46.259910 \mathrm{Ah}$ & $46.565840 \mathrm{Ah}$ \\
\hline $45^{\circ} \mathrm{C}$ & $45.454770 \mathrm{Ah}$ & $45.279540 \mathrm{Ah}$ & $45.220800 \mathrm{Ah}$ & $45.382554 \mathrm{Ah}$ & $45.654083 \mathrm{Ah}$ \\
\hline
\end{tabular}

Table.7 shows the discharge capacity.

Tab.7 Discharging capacity

\begin{tabular}{|l|l|l|l|l|c|}
\hline Temperature $\backslash$ multiplie & $0.2 \mathrm{C} \approx 9 \mathrm{~A}$ & $0.444 \mathrm{C} \approx 20 \mathrm{~A}$ & $0.6 \mathrm{C} \approx 27 \mathrm{~A}$ & $0.8 \mathrm{C} \approx 36 \mathrm{~A}$ & $1 \mathrm{C} \approx 45 \mathrm{~A}$ \\
\hline
\end{tabular}




\begin{tabular}{|c|c|c|c|c|c|}
\hline $\mathrm{r}$ & & & & & \\
\hline$-20^{\circ} \mathrm{C}$ & $31.432045 \mathrm{Ah}$ & $31.364556 \mathrm{Ah}$ & $32.044350 \mathrm{Ah}$ & $33.496312 \mathrm{Ah}$ & $35.160382 \mathrm{Ah}$ \\
\hline $0^{\circ} \mathrm{C}$ & $42.542468 \mathrm{Ah}$ & $42.132643 \mathrm{Ah}$ & $42.143060 \mathrm{Ah}$ & $42.136828 \mathrm{Ah}$ & $42.133495 \mathrm{Ah}$ \\
\hline $10^{\circ} \mathrm{C}$ & $44.925846 \mathrm{Ah}$ & $44.695048 \mathrm{Ah}$ & $44.634696 \mathrm{Ah}$ & $44.664065 \mathrm{Ah}$ & $44.636371 \mathrm{Ah}$ \\
\hline $25^{\circ} \mathrm{C}$ & $46.400115 \mathrm{Ah}$ & $46.590549 \mathrm{Ah}$ & $46.243173 \mathrm{Ah}$ & $46.263523 \mathrm{Ah}$ & $46.250023 \mathrm{Ah}$ \\
\hline
\end{tabular}

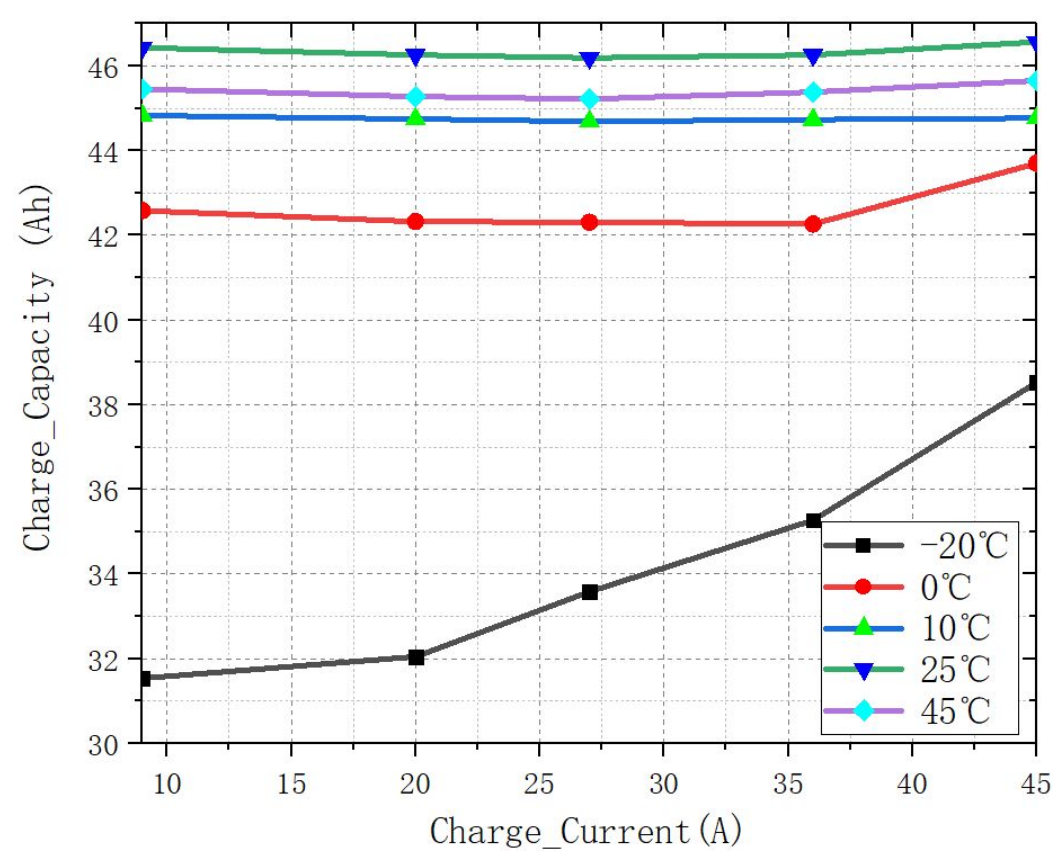

Fig. 11 Charging Capacity at Different Temperatures and Rates

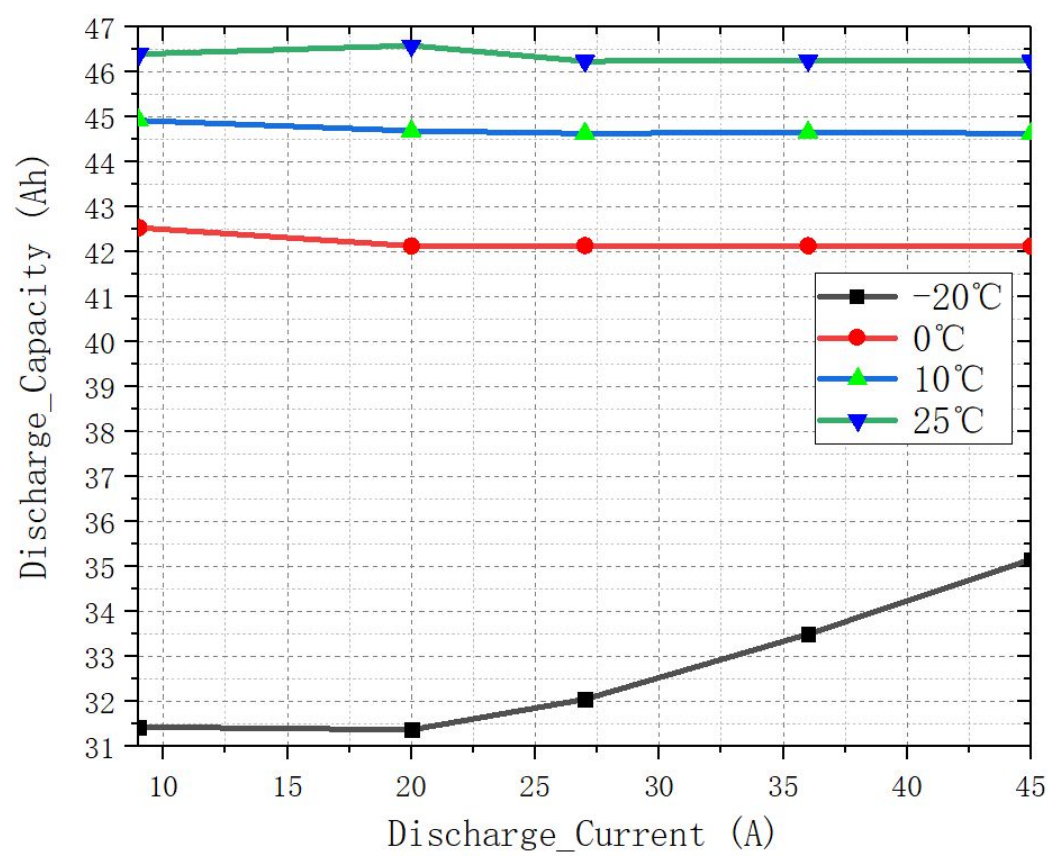


Fig. 12 Discharging Capacity at Different Temperatures and Rates

In the process of temperature decreasing gradually, the charge-discharge capacity has obvious decay phenomenon. Especially when the temperature is -20 degrees, the charge-discharge capacity of the battery decreases significantly, only about $75 \%$ of the standard capacity. Therefore, the available capacity of battery must be calibrated when estimating SOC in such extreme low temperature environment. In addition, after the double discharge capacity test at the same temperature, the discharge capacity at different rates in the first stage will vary greatly, with the maximum discharge capacity not exceeding $2 \mathrm{Ah}$, while the total discharge capacity of the two discharge stages at different rates is similar, not exceeding 0.5Ah. What's more, the capacity test results of different Charge-discharge current at low temperature $\left(-20^{\circ} \mathrm{C}\right.$ and $\left.0{ }^{\circ} \mathrm{C}\right)$ are significantly different from that at normal temperature, especially at $-20^{\circ} \mathrm{C}$, when the battery is loaded with larger Charge-discharge current, the Charge-discharge capacity is significantly higher. It is possible that the phenomenon of battery at low temperature is due to the inhibition of low temperature on the electrochemical activity of battery and the increase of battery temperature when the battery is charged and discharged at high current. Therefore, the combination of the two condition makes the capacity of battery charged and discharged at high rate higher at $-20{ }^{\circ} \mathrm{C}$. When used in extreme low temperature environment, the actual available capacity of lithium-ion batteries can be effectively corrected, then the industry problem of large SOC estimation error of lithiumion batteries in low temperature environment has been solved to a certain extent. It can effectively predict the actual available capacity under the current average discharge rate according to the actual discharge rate of the battery and improve the estimation accuracy of SOC in a high discharge rate.

\subsection{Verification Experiments}

Firstly, the original SOC estimation model based on Ampere-hour method, the improved SOC estimation model based on open-circuit voltage method to correct initial error and an adaptive improved SOC estimation model based on Ampere-hour method are built in SIMULINK respectively. Secondly, in order to verify SOC estimation effects of the adaptive improved Ampere-hour method under different conditions, specific verification experiment was designed. At room temperature, the simulation experiment of high rate discharge of battery after aging experiment was designed in section 3.5.1, compared with the traditional improved Ampere-hour method, the optimization effect of capacity attenuation factor $\mathrm{k}$ and capacity correction on SOC estimation was verified. Another experiment was designed to simulate the working condition of an Electric Vehicle in extreme low temperature for one day in section 3.5.2, and the results of the adaptive improved ampere-hour method considering the temperature effect were verified by comparing with those of traditional improved method without considering the temperature effect. Finally, in order to further illustrate the adaptability of the adaptive improved Ah algorithm, in the case of SOC initial error, Beijing 
Bus Dynamic Stress Test (BBDST) working condition experiment was designed in section 3.5.3, and the SOC estimation effect of this algorithm was compared with Extended Kalman Filter algorithm.

\subsubsection{Adaptability to Battery Aging and Large Rate Discharge Conditions}

The double constant current discharge validation experiment of $1 \mathrm{C}$ and $0.1 \mathrm{C}$ was carried out after the working condition experiment of battery. After the battery has been tested for a period of time, the following statistical methods are used to standardize the number of battery cycle charge and discharge, and the number of battery cycle charge and discharge is about 285.

(1) After the battery capacity is calibrated, initialize the counter and set it to zero,

(2) The effect of discharge to the counter. In case of single discharge (from the end of last charge to the beginning of next charge), the change of SOC less than 0.1 will not be counted; if it is more than 0.1 , the counter value will increase by half of the discharge,

(3) The effect of charging to the counter. In case of single charge (during a single charge, the battery can be put aside, but can't be discharged for use), the SOC change less than 0.1 will not be counted; if the SOC change is more than 0.1 , the counter value will increase half of the charge amount.

The current and voltage of the experiment are shown in Fig. 13.

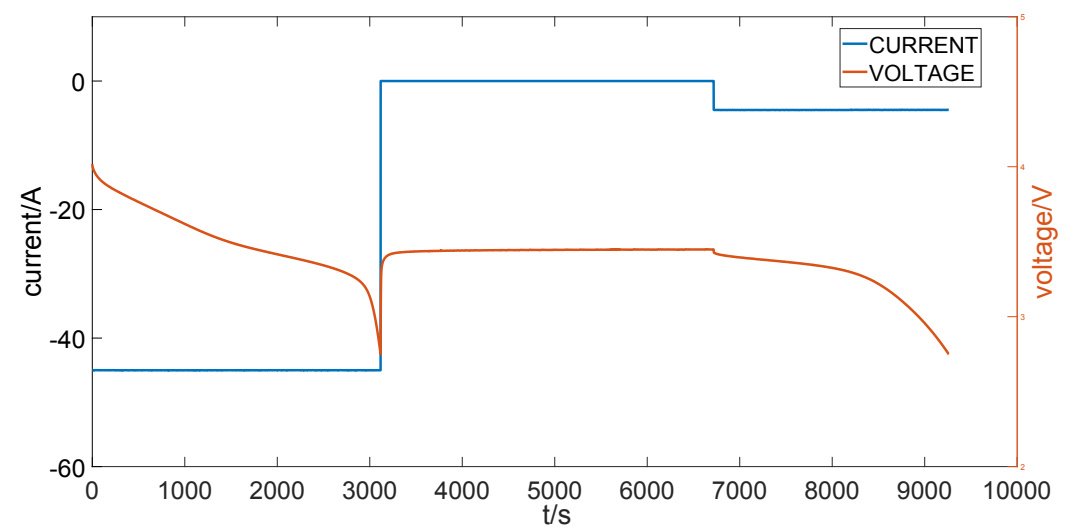

Fig. 13 Double discharge experimental current and voltage

Firstly, the current, voltage and capacity data of double discharge verification experiments are derived, then real-time estimation of SOC is carried out in SOC estimation modules. The estimation errors of the two methods are analyzed by comparing the SOC estimation results of traditional Ampere-hour method, adaptive improved Ampere-hour method and the experimental data, as shown in Fig. 14 and Fig. 15. 


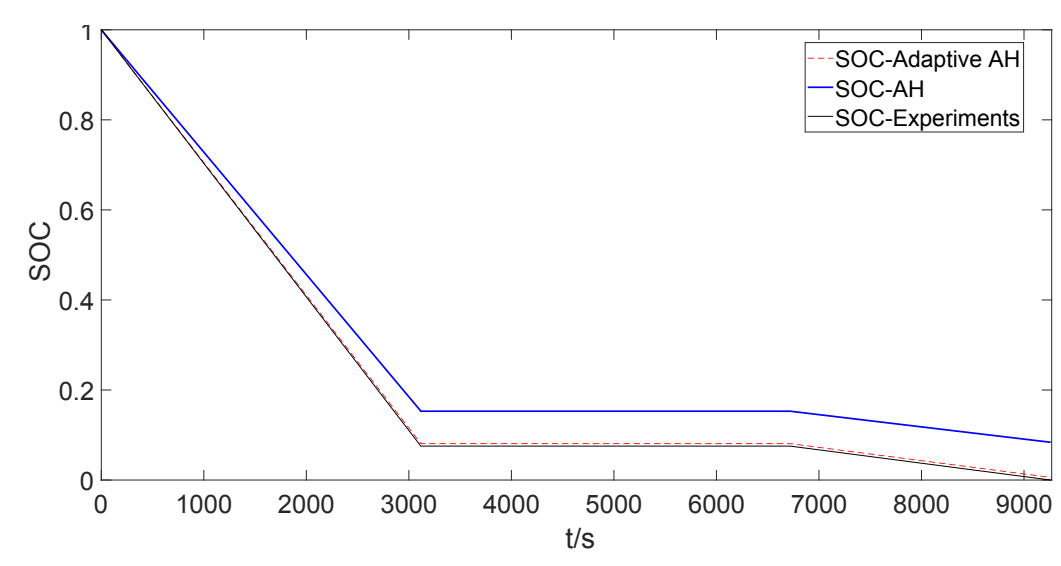

Fig. 14 SOC Estimation Effect

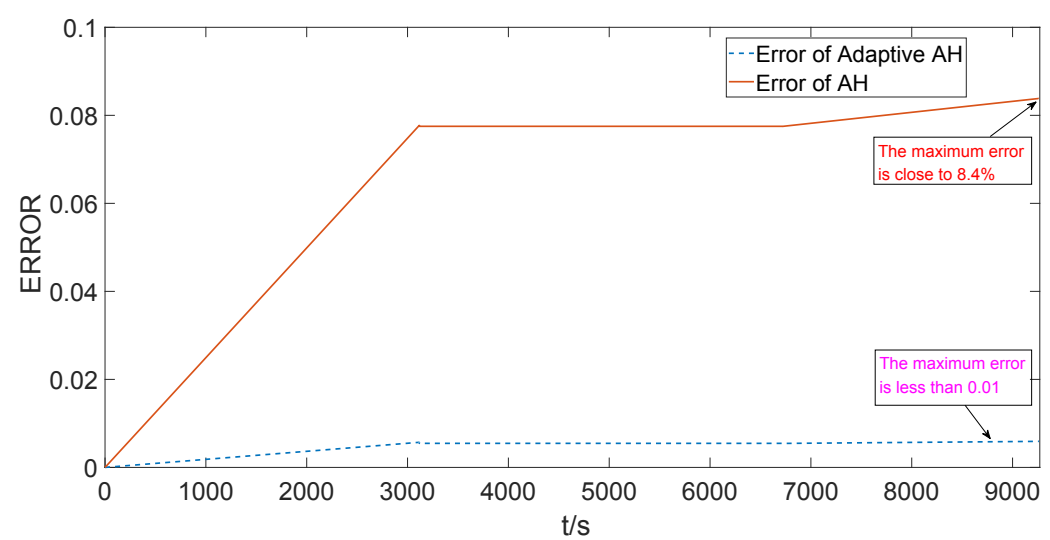

Fig. 15 Error of SOC Estimation

According to the capacity change data of constant current discharge verification experiment, the actual capacity decay of battery after 285 cycles of Charge-discharge is about $91 \%$ of the initial capacity. At this time, the calculated value of capacity decay factor $\mathrm{k}$ in Adaptive Ah method is about 0.925 , which accurately describes the capacity decay phenomenon caused by battery aging. According to Fig. 14 and Fig. 15, considering the effect of battery cycle times and discharge rate on the available capacity of batteries, compared with the original Ampere-hour method, the adaptive improved Ampere-hour method achieves better estimation results. The largest estimation error is less than 0.01 , while the maximum error of the traditional Ah method is close to 0.084 . At the same time, it can be clearly observed that the SOC estimation error of the traditional Ampere-hour method presents a growing trend, which is the most remarkable feature of the open-loop estimation method, but the adaptive improved Ah method can effectively solve the error accumulation of the Ampere-hour method by continually modifying the initial value of SOC so that the SOC estimation value is always near the real value.

\subsubsection{Adaptability at Extreme Temperature}

An experiment was designed to simulate the working condition of an Electric Vehicle driving for one day at a extreme low temperature environment (-10 degrees). The battery has been charged with constant current and constant 
voltage for 8 hours before the experiment. The specific experimental process is as follows:

(1) Running three times under DST conditions and then shelving for 4 hours.

(2) Running DST twice and shelving for 35 minutes.

(4) Running DST twice and shelving for 4 hours.

(5) Three times of DST operation, and then shelf for 2 hours, the first five steps of environmental temperature are 10 degrees.

(6) Constant current and constant voltage are charged and put aside for 8 hours. The ambient temperature of this process is controlled at 10 degrees, and the end is achieved.

Fig. 16 shows the current and voltage curves of batteries during DST test under a certain SOC condition, and Fig. 17 shows the current of simulating a day's operation test of an Electric Vehicle.

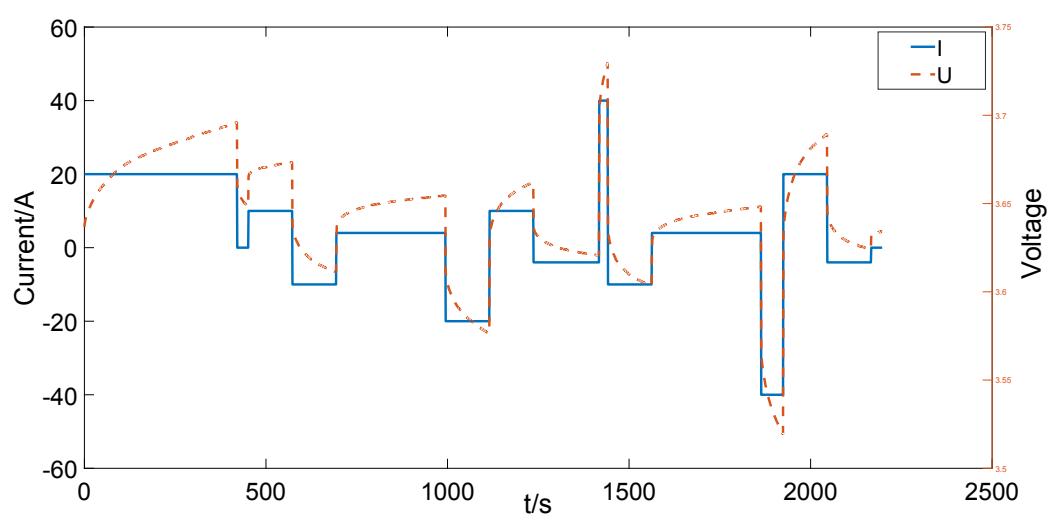

Fig. 16 Current-Voltage in Single DST Working Conditions

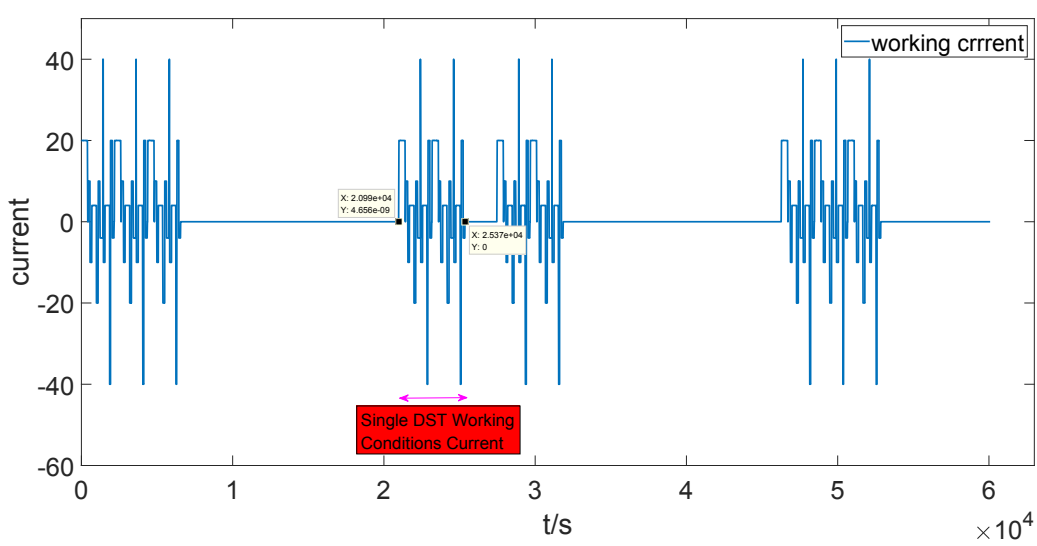

Fig. 17 Simulated Operating Current for One Day

The experimental parameters needed for SOC estimation include operating temperature (- 10 degrees) and current, average temperature (- 10 degrees or 10 degrees) in shelf stage, shelf time and voltage. The parameters that need to be given as follows: initial SOC before the experiment and the number of cycles of Charge-discharge. The above data are imported into the SOC estimation module, and the experimental results shown in Fig. 18 and Fig. 19 are obtained. 


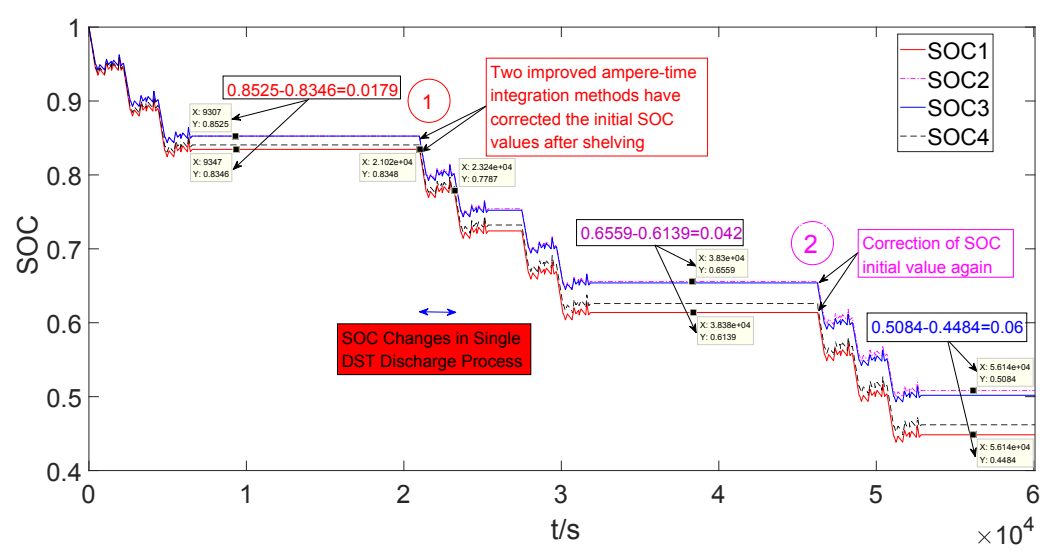

Fig. 18 SOC Estimation at -10 Degrees

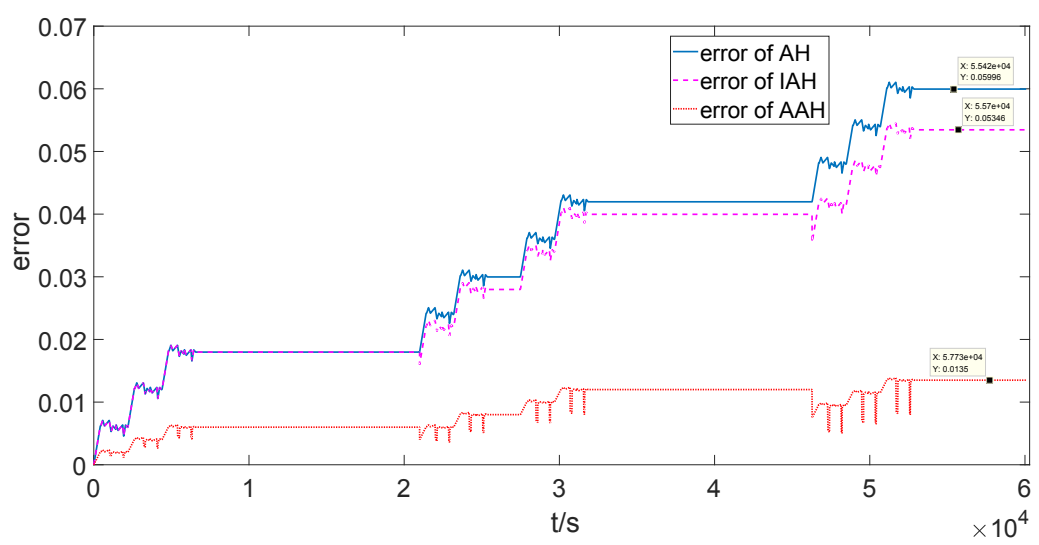

Fig. 19 Error of SOC Estimation at -10 Degrees

Fig. 18 is a comparison between the estimated results of three SOC estimation methods and the actual SOC change curves, in which SOC1 is the actual SOC change curve, which is drawn by verifying the experimental data. SOC2 is the SOC estimation obtained by the traditional Ampere-hour simulation model, SOC3 is the SOC estimation obtained by the improved Ah method simulation model, and SOC4 is the SOC estimation obtained by the adaptive improved ampere-hour method simulation model. Observe curves SOC3 and SOC4, in the position of (1) and (2) in Fig. 18, the SOC values are revised by looking up tables. The revised SOC3 and SOC4 curves are closer to the true curve SOC1, while the estimated error of the unmodified traditional Ampere-hour Method gradually increases from 0.0179 to 0.06 .

The three curves in Figure.19 represent the errors of the three estimates in turn. The results show that the maximum error of SOC estimation is about $1.35 \%$ by the adaptive improved Ampere-hour method, while the error of the improved Ampere-hour method and the original Ampere-hour method is more than 5\%, which shows the applicability of this method to extreme temperature working conditions.

\subsubsection{Adaptability of initial SOC error}

Limited by the influence of current sampling accuracy, the cumulative error caused by integration will seriously 
affect SOC estimation accuracy when the traditional Ah method was used. In order to solve the common problem of Ah method, two kinds of SOC initial value correction algorithms are designed for different working conditions by the adaptive improved Ah algorithm. When the BMS downtime meets the condition of enabling the OCV method, the SOC initial value will be accurately corrected based on accurate OCV-SOC mapping relationship. When the downtime can't meet the condition of using the OCV method for a long time (more than 48 hours), based on the internal resistance model of LIB, the estimated OCV value is calculated by using the closed-circuit voltage method, and then the initial value of SOC is corrected by using the OCV-SOC relationship. In order to verify the performance of adaptive improved Ah method in the case of initial SOC error, the BBDST working condition verification experiment was designed at room temperature, and the SOC estimation effect of this algorithm was compared with EKF algorithm. The BBDST working condition and its SOC estimation results are shown in the figure 20:

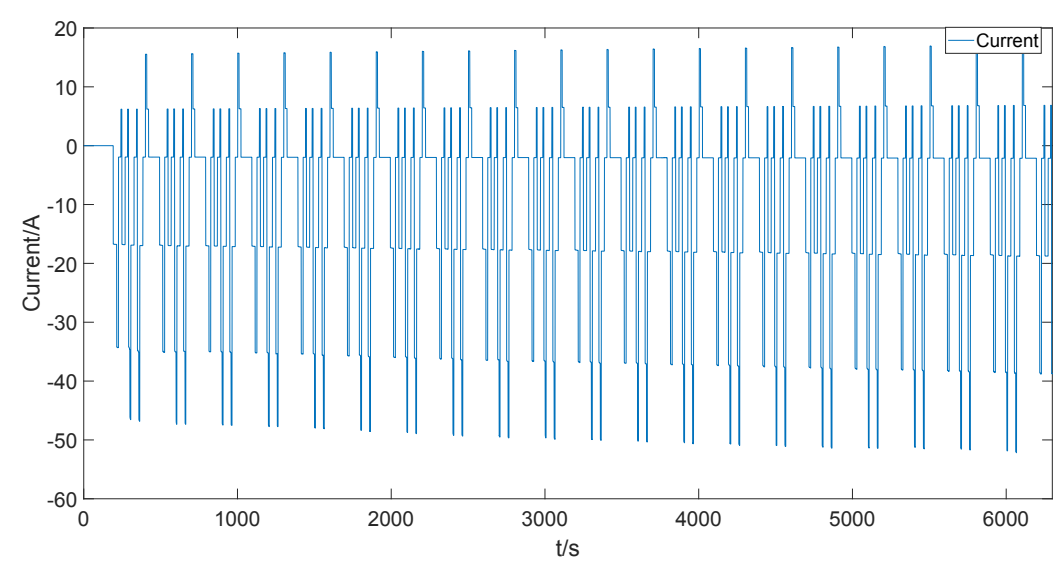

Fig. 20 (a) BBDST working condition

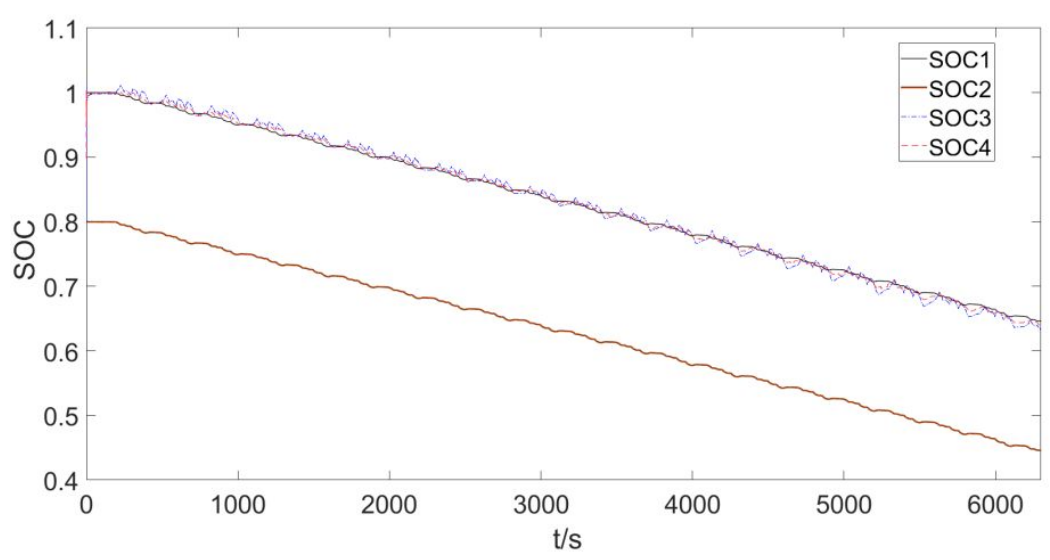

Fig. 20 (b) SOC estimation results under BDDST 


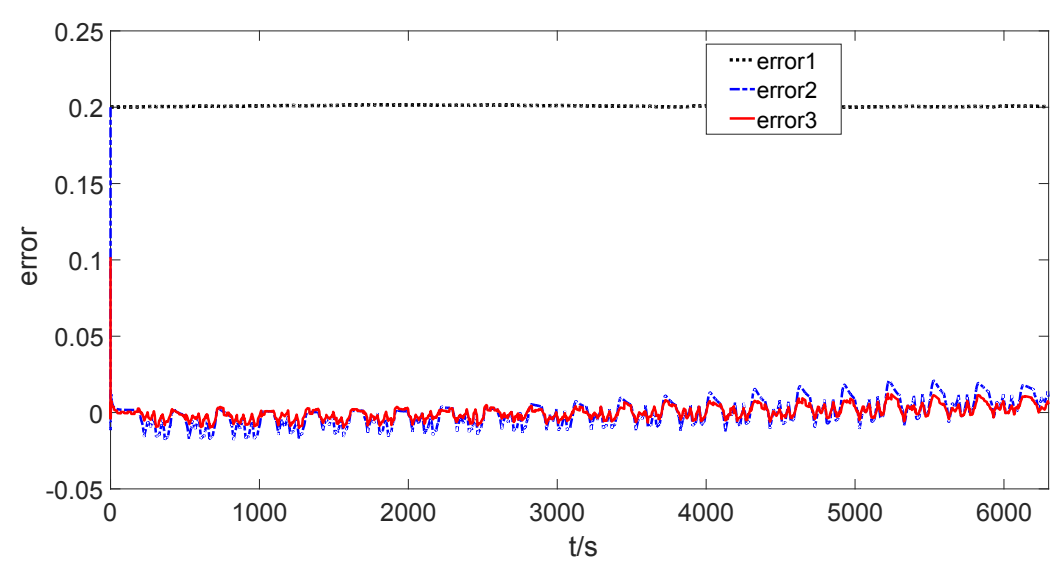

Fig. 20 (c) SOC estimation error under BDDST

Figure 20 (b) shows the estimation results of three SOC estimation algorithms under the working condition of BBDST shown in Figure 20 (a), where SOC1 is the real SOC curve, SOC2 is the SOC estimation effect of traditional Ah method, it can be seen that the initial SOC error seriously affects SOC estimation accuracy of Ah method, and the initial error can't be eliminated by Ah method. SOC3 and SOC4 are the SOC estimation results of EKF algorithm and the adaptive improved Ah method respectively, from the SOC estimation curve of figure 20 (b) and the SOC error curve of figure 20 (c), it can be found that the two methods obviously restrain the initial error of SOC, and the estimation results fluctuate near the true value of SOC1, and the estimation effect of proposed algorithm is better than that of EKF algorithm to some extent. Furthermore, compared with EKF algorithm, the adaptive improved Ah method is mainly used for SOC estimation in two ways: cumulative summation (integration) and linear look-up table, it does not include complex matrix multiplication and inversion, which greatly reduces the computational complexity and proves the adaptability and scientific of the proposed adaptive improved Ah algorithm.

\section{Conclusions}

Based on traditional Ah Method, the influence of three main parameters, temperature, Charge-discharge current and the number of cyclic charges and discharges, on the accuracy of the Ah method in SOC estimation is analyzed. The capacity attenuation factor $\mathrm{k}$ and capacity composite correction factor $\mathrm{F}$ are innovatively proposed, which simplifies and quantifies the parameter changes in Ah algorithm caused by external factors. The relationship between parameter variation and external factors is discussed, and a novel adaptive improved Ah algorithm is proposed. The SOC estimation model is built in SIMULINK, and the validation experiments of the algorithm under three typical conditions are designed, results show that the estimation error of adaptive improved method is less than $2 \%$, which proves the adaptability of proposed algorithm to typical working 
conditions. Result of BBDST shows that when there is initial SOC error, the algorithm can effectively suppress the error and realize the accurate estimation of SOC, which further proves the adaptive ability of the algorithm. In the future, in the field of improving SOC estimation accuracy of Ah algorithm, the influence mechanism of more working conditions should be studied, and balance the contradiction between algorithm performance and calculation complexity.

5. Acknowledgments

The work was supported by National Natural Science Foundation of China (No. 61801407).

6. Reference

1. Zhao, L., G. Ji, and Z. Liu, Design and Experiment of Nonlinear Observer with Adaptive Gains for Battery State of Charge Estimation. Energies, 2017. 10(12): p. 2046.

2. Mathew, M., et al., Comparative Analysis of Lithium-Ion Battery Resistance Estimation Techniques for Battery Management Systems. Energies, 2018. 11(6): p. 1490.

3. Zhang, Z., et al., SOC Estimation of Lithium-Ion Battery Pack Considering Balancing Current. IEEE Transactions on Power Electronics, 2018. 33(3): p. 2216-2226.

4. Zhang, Y., et al., Parameters Identification and Sensitive Characteristics Analysis for Lithium-Ion Batteries of Electric Vehicles. Energies, 2017. 11(1): p. 19.

5. Zhang, R., et al., State of the Art of Lithium-Ion Battery SOC Estimation for Electrical Vehicles. Energies, 2018. 11(7): p. 1820.

6. Yang, D., et al., State-of-health estimation for the lithium-ion battery based on support vector regression. Applied Energy, 2018. 227: p. 273-283.

7. Xia, B., et al., Strong Tracking of a H-Infinity Filter in Lithium-Ion Battery State of Charge Estimation. Energies, 2018. 11(6): p. 1481.

8. Meng, J., et al., Overview of Lithium-Ion Battery Modeling Methods for State-of-Charge Estimation in Electrical Vehicles. Applied Sciences, 2018. 8(5): p. 659.

9. Long, Z., et al.,Massive battery pack data compression and reconstruction using a frequency division model in battery management systems. Journal of Energy Storage. 2020. 28: p. 101.

10. $\mathrm{Wu}, \mathrm{J} ., \mathrm{C}$. Zhang, and Z. Chen, An online method for lithium-ion battery remaining useful life estimation using importance sampling and neural networks. Applied Energy, 2016. 173: p. 134-140.

11. Xin, L., C. A hybrid state-of-charge estimation method based on credible increment for electric vehicle applications with large sensor and model errors. Journal of Energy Storage, 2020. 27: p. 101-106. 
12. Wu, J., et al., Energy Management Strategy for Grid-Tied Microgrids Considering the Energy Storage Efficiency. IEEE Transactions on Industrial Electronics, 2018. 65(12): p. 9539-9549.

13. Wu, J., et al., A novel state of health estimation method of Li-ion battery using group method of data handling. Journal of Power Sources, 2016. 327: p. 457-464.

14. Wei, Z., et al., Comparative study of methods for integrated model identification and state of charge estimation of lithium-ion battery. Journal of Power Sources, 2018. 402: p. 189-197.

15. Wei, Z., et al., Online State of Charge and State of Health Estimation for a Lithium-Ion Battery Based on a DataModel Fusion Method. Energies, 2018. 11(7): p. 1810.

16. Wei, J., et al., System state estimation and optimal energy control framework for multicell lithium-ion battery system. Applied Energy, 2017. 187: p. 37-49.

17. Wang, S., et al., Online dynamic equalization adjustment of high-power lithium-ion battery packs based on the state of balance estimation. Applied Energy, 2016. 166: p. 44-58.

18. Wang, W., et al., Unscented Kalman Filter-Based Battery SOC Estimation and Peak Power Prediction Method for Power Distribution of Hybrid Electric Vehicles. IEEE Access, 2018. 6: p. 35957-35965.

19. Wang, S., et al., The parameter identification method study of the splice equivalent circuit model for the aerial lithium-ion battery pack. Measurement and Control, 2018. 51(5-6): p. 125-137.

20. Lim, D.-J., et al., A Mixed SOC Estimation Algorithm with High Accuracy in Various Driving Patterns of EVs. Journal of Power Electronics, 2016. 16(1): p. 27-37.

21. Wang, S., et al., A novel safety anticipation estimation method for the aerial lithium-ion battery pack based on the real-time detection and filtering. Journal of Cleaner Production, 2018. 185: p. 187-197.

22. Tian, J., R. Xiong, and Q. Yu, Fractional-Order Model-Based Incremental Capacity Analysis for Degradation State Recognition of Lithium-Ion Batteries. IEEE Transactions on Industrial Electronics, 2019. 66(2): p. 1576-1584.

23. Sturm, J., et al., State estimation of lithium-ion cells using a physicochemical model based extended Kalman filter. Applied Energy, 2018. 223: p. 103-123.

24. Li, Z., et al., Comparison of methods for improving SOC accuracy through an ampere-hour integration approach. Tsinghua Uinv(Sci \& Tech), 2010. 50(8): p. 1293-1296.

25. Liu, Z., X. Dang, and B. Jing, A Novel Open-circuit Voltage Based State of Charge Estimation for Lithium-Ion Battery by Multi-Innovation Kalman Filter. IEEE Access, 2019. 7: p. 49432-49447.

26. Pei, F., et al., Real-time estimation of current conversion and discharge characteristics and state of charge of 
electric vehicle power battery. Chinese Journal of electrical engineering, 2005. 9: 164-168.

27. Shehab El Din, M., A.A. Hussein, and M.F. Abdel-Hafez, Improved Battery SOC Estimation Accuracy Using a Modified UKF With an Adaptive Cell Model Under Real EV Operating Conditions. IEEE Transactions on Transportation Electrification, 2018. 4(2): p. 408-417.

28. Feng, F., et al., A low temperature SOC estimation algorithm for lithium ion batteries. Journal of electrical technology, 2014. 29(07): p. 53-58.

29. Zhao, L., M. Lin, and Y. Chen, Least-squares based coulomb counting method and its application for state-of-charge (SOC) estimation in electric vehicles. International Journal of Energy Research, 2016. 40(10): p. 1389-1399.

30. Feng, F., R. Lu, and C. Zhu, A Combined State of Charge Estimation Method for Lithium-Ion Batteries Used in a Wide Ambient Temperature Range. Energies, 2014. 7(5): p. 3004-3032.

31. Ma, Y., et al., Equalization of Lithium-Ion Battery Pack Based on Fuzzy Logic Control in Electric Vehicle. IEEE Transactions on Industrial Electronics, 2018. 65(8): p. 6762-6771.

32. Luo, M., et al., Ternary-material lithium-ion battery SOC estimation under various ambient temperature. Ionics, 2018. 24(7): p. 1907-1917.

33. Liu, Z., X. Dang, and H. Sun, Online State of Charge Estimation for Lithium-Ion Battery by Combining Incremental Autoregressive and Moving Average Modeling with Adaptive H-Infinity Filter. Mathematical Problems in Engineering, 2018. 2018: p. 1-16.

34. Lee, K.-T., M.-J. Dai, and C.-C. Chuang, Temperature-Compensated Model for Lithium-Ion Polymer Batteries With Extended Kalman Filter State-of-Charge Estimation for an Implantable Charger. IEEE Transactions on Industrial Electronics, 2018. 65(1): p. 589-596.

35. Lai, X., Y. Zheng, and T. Sun, A comparative study of different equivalent circuit models for estimating state-of-charge of lithium-ion batteries. Electrochimica Acta, 2018. 259: p. 566-577.

36. Hu, X., D. Cao, and B. Egardt, Condition Monitoring in Advanced Battery Management Systems: Moving Horizon Estimation Using a Reduced Electrochemical Model. IEEE/ASME Transactions on Mechatronics, 2018. 23(1): p. 167-178.

37. Li, Z., et al., Temperature characteristics of power lithium iron phosphate battery. Journal of mechanical engineering, 2011. 47(18): p. 115-120.

38. Wu, X., X. Li, and J. Du, State of Charge Estimation of Lithium-Ion Batteries Over Wide Temperature Range Using Unscented Kalman Filter. IEEE Access, 2018. 6: p. 41993-42003. 
39. Afshari, H.H., et al., Reliable state of charge and state of health estimation using the smooth variable structure filter. Control Engineering Practice, 2018. 77: p. 1-14.

40. Zhang, Y., et al., A novel model of the initial state of charge estimation for LiFePO4 batteries. Journal of Power Sources, 2014. 248: p. 1028-1033.

41. Xing, Y., et al., State of charge estimation of lithium-ion batteries using the open-circuit voltage at various ambient temperatures. Applied Energy, 2014. 113: p. 106-115.

42. Osaka, T., et al., Influence of capacity fading on commercial lithium-ion battery impedance. Journal of Power Sources, 2003. 119-121: p. 929-933.

43. Sun, F., R. Xiong, and H. He, Estimation of state-of-charge and state-of-power capability of lithium-ion battery considering varying health conditions. Journal of Power Sources, 2014. 259: p. 166-176.

44. Lee, S., et al., State-of-charge and capacity estimation of lithium-ion battery using a new open-circuit voltage versus state-of-charge. Journal of Power Sources, 2008. 185(2): p. 1367-1373.

45. Ma, Y., et al., Remaining Useful Life Prediction of Lithium-Ion Battery Based on Gauss-Hermite Particle Filter. IEEE Transactions on Control Systems Technology, 2019. 27(4): p. 1788-1795.

46. Hongwen, H., et al., State-of-Charge Estimation of the Lithium-Ion Battery Using an Adaptive Extended Kalman Filter Based on an Improved Thevenin Model. IEEE Transactions on Vehicular Technology, 2011. 60(4): p. 1461-1469.

47. Chen, Z., et al., Online state of charge estimation of Li-ion battery based on an improved unscented Kalman filter approach. Applied Mathematical Modelling, 2019. 70: p. 532-544.

48. Shen, P., et al., The Co-estimation of State of Charge, State of Health, and State of Function for Lithium-Ion Batteries in Electric Vehicles. IEEE Transactions on Vehicular Technology, 2018. 67(1): p. 92-103. 